\title{
Innate immune sensing of bacterial modifications of Rho GTPases by the Pyrin inflammasome
}

Hao Xu ${ }^{1 *}$, Jieling Yang ${ }^{1,2 *}$, Wenqing Gao ${ }^{1}$, Lin $\mathrm{Li}^{1}$, Peng $\mathrm{Li}^{1}$, Li Zhang ${ }^{1}$, Yi-Nan Gong ${ }^{1}$, Xiaolan Peng ${ }^{1}$, Jianzhong Jeff Xi ${ }^{3}$, She $\mathrm{Chen}^{1}$, Fengchao Wang $^{1} \&$ Feng Shao ${ }^{1,2,4}$

Cytosolic inflammasome complexes mediated by a pattern recognition receptor (PRR) defend against pathogen infection by activating caspase 1. Pyrin, a candidate PRR, can bind to the inflammasome adaptor ASC to form a caspase 1-activating complex ${ }^{1,2}$. Mutations in the Pyrin-encoding gene, $M E F V$, cause a human autoinflammatory disease known as familial Mediterranean fever $^{3-5}$. Despite important roles in immunity and disease, the physiological function of Pyrin remains unknown. Here we show that Pyrin mediates caspase 1 inflammasome activation in response to Rho-glucosylation activity of cytotoxin $\mathrm{TcdB}^{6-8}$, a major virulence factor of Clostridium difficile, which causes most cases of nosocomial diarrhoea. The glucosyltransferaseinactive TcdB mutant loses the inflammasome-stimulating activity. Other Rho-inactivating toxins, including FIC-domain adenylyltransferases (Vibrio parahaemolyticus VopS and Histophilus somni IbpA) and Clostridium botulinum ADP-ribosylating C3 toxin, can also biochemically activate the Pyrin inflammasome in their enzymatic activitydependent manner. These toxins all target the Rho subfamily and modify a switch-I residue. We further demonstrate that Burkholderia cenocepacia inactivates RHOA by deamidating Asn 41, also in the switch-I region, and thereby triggers Pyrin inflammasome activation, both of which require the bacterial type VI secretion system (T6SS). Loss of the Pyrin inflammasome causes elevated intra-macrophage growth of $B$. cenocepacia and diminished lung inflammation in mice. Thus, Pyrin functions to sense pathogen modification and inactivation of Rho GTPases, representing a new paradigm in mammalian innate immunity.

C. difficile is the major cause of hospital-acquired infectious diarrhoea and antibiotic-associated pseudomembranous colitis. The major virulence factors of $C$. difficile are two secreted protein toxins (TcdA and $\mathrm{TcdB})^{7,8}$. TcdA/B and the related Clostridium sordellii lethal toxin (TcsL) belong to the large clostridial glucosylating cytotoxin family that inactivates members of Rho and/or Ras-family small GTPases by monoglucosylating a threonine residue critical for GTP binding ${ }^{6}$. Recent studies indicate that $\mathrm{Tcd} A / \mathrm{B}$ can activate the inflammasome $\mathrm{e}^{9,10}$. Consistently, recombinant $\mathrm{TcdB}$ triggered robust caspase 1 activation, interleukin (IL) $-1 \beta$ production and pyroptosis in primary bone marrow-derived macrophages (BMDMs) (Fig. 1a and Extended Data Fig. 1a-d). TcsL, sharing $85 \%$ sequence homology to $\mathrm{TcdB}$, showed no such activities. As expected, both TcdB and TcsL caused cell rounding, which did not occur with the glucosyltransferase-deficient TcdB(W102A/D288N) and $\mathrm{TcsL}\left(\mathrm{D} 286 \mathrm{~N} / \mathrm{D} 288 \mathrm{~N}\right.$ ) mutants ${ }^{6}$ (referred to as $\mathrm{TcdB}^{\mathrm{m}}$ and $\mathrm{TcsL}^{\mathrm{m}}$, respectively) (Extended Data Fig. $2 \mathrm{a}$ ). $\mathrm{TcdB}^{\mathrm{m}}$ could not induce caspase 1 inflammasome activation (Fig. 1a and Extended Data Fig. 1a-d). Thus, the Rho-glucosylating activity of TcdB but not TcsL triggers inflammasome activation in mouse macrophages.

The PYRIN-CARD domain adaptor ASC is critical for caspase 1 activation mediated by a PYRIN-domain PRR. Consistent with previous studies $^{9,10}$, inflammasome activation in Asc (also known as Pycard) ${ }^{-/-}$
BMDMs was resistant to TcdB stimulation (Fig. 1a and Extended Data Fig. 1c-d). By contrast, Nlrp3 $3^{-1-} N l r c 4^{-1-}$ and Aim $2^{-1-}$ BMDMs remained sensitive to TcdB. As a control, enterohaemorrhagic Escherichia coli (EHEC) type III secretion needle protein EprI induced little IL-1 $\beta$ production in $\mathrm{Nlrp3} 3^{-1-} \mathrm{Nlrc} 4^{-1-}$ and $\mathrm{Asc}^{-1-}$ BMDMs, whereas pyroptosis was only diminished in Nlrp3 $3^{-1-} \mathrm{Nlrc} 4^{-1-}$ BMDMs. Deletion of Nod1/2, involved in sensing bacterial activation of Rho GTPases in the NF- $\kappa B$ pathway ${ }^{11,12}$, did not affect TcdB-induced caspase 1 activation (Extended Data Fig. 1e-g). TcdB induced similar caspase 1 activation in BMDMs from different mouse inbred strains (Extended Data

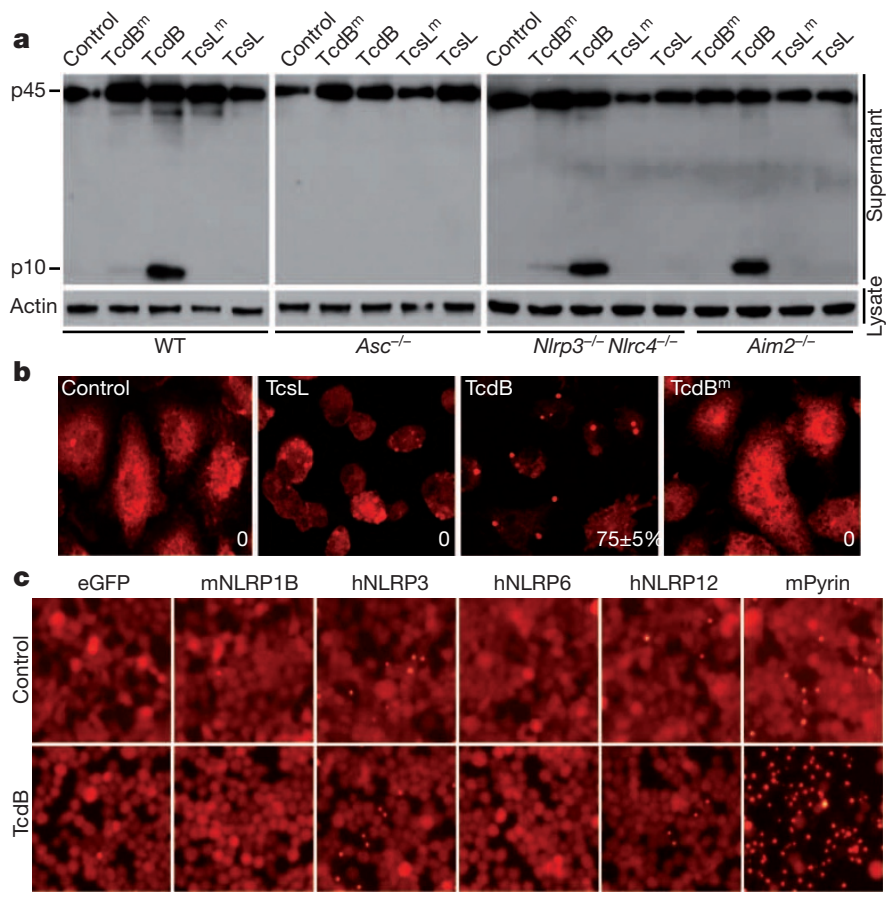

Figure 1 Inflammasome activation by TcdB and identification of Pyrin as the candidate immune sensor. a, Assays of inflammasome activation by TcdB and TcsL in BMDMs from wild-type (WT, C57BL/6) or indicated knockout mice. Macrophage supernatants were collected for anti-caspase 1 immunoblotting (p45, pro-caspase 1; p10, mature caspase 1). b, Anti-ASC immunostaining of TcdB- and TcsL-stimulated BMDMs. Percentages of cells showing the ASC foci are marked. c, Assays of different PYRIN- or CARDdomain proteins in supporting TcdB-induced ASC foci formation in 293T cells stably expressing RFP-ASC. mNLRP1B, mouse NLRP1B; hNLRP3/6/12, human NLRP3/6/12; mPyrin, mouse Pyrin. TcdB ${ }^{\mathrm{m}}$ and $\mathrm{TcsL}^{\mathrm{m}}$ denote the glucosyltransferase-deficient TcdB(W102A/D288N) and TcsL(D286N/ D288N) mutants, respectively. Data in all panels are representative of at least three repetitions.

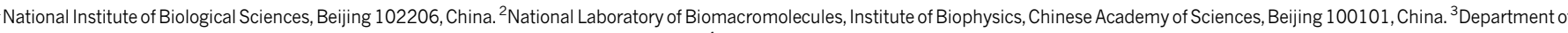

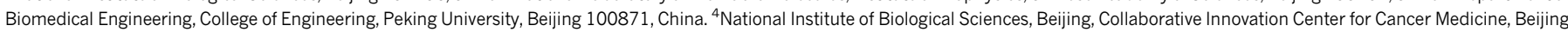
102206, China.

*These authors contributed equally to this work. 
Fig. 1h). TcdB, but not $\mathrm{TcdB}^{\mathrm{m}}$ or TcsL, rendered endogenous ASC aggregation (foci formation) in BMDMs (Fig. 1b). Thus, TcdB activates an ASC-containing inflammasome independently of NLRP3, NLRC4, AIM2 and NOD1/2.

Red fluorescent protein (RFP)-ASC foci formation reconstituted in 293T cells was used to screen for the upstream PRR protein in sensing TcdB. Of a total of ten PYRIN-domain proteins tested (NLRP2, NLRP3, NLRP5, NLRP6, NLRP7, NLRP8, NLRP9, NLRP11, NLRP12 and Pyrin), only Pyrin supported TcdB-induced RFP-ASC foci formation (Fig. 1c and data not shown). Mutation of Pyrin-encoding $M E F V$ causes the human autoinflammatory disorder familial Mediterranean fever (FMF) ${ }^{3,4}$. Consistently, Pyrin has been shown to interact with ASC through their amino-terminal PYRIN domains to promote caspase 1 activation in vitro ${ }^{1,2,13}$. FMF-causing mutations are gain-of-function and the disease-like symptom in the knock-in mice results from ASC-dependent excessive IL- $1 \beta$ release $^{5}$. Pyrin is primarily expressed in monocytes and dendritic cells. We noticed that, in contrast to primary BMDMs, immortalized mouse BMDMs (iBMDMs), primary bone marrowderived dendritic cells and immortalized mouse DC2.4 dendritic cells showed no inflammasome response to TcdB stimulation (Fig. 2a). Mefv messenger RNA was not detectable in these three cell types (Fig. 2b). Stable expression of mouse or human Pyrin but not NLRP3 in DC2.4 cells rendered robust inflammasome responses to TcdB without increasing the sensitivity to the NAIP inflammasome agonist (Shigella flexneri type III secretion needle protein $\mathrm{MxiH}$ ) and the NLRP3 inflammasome agonist lipopolysaccharide (LPS) plus nigericin (Fig. $2 \mathrm{c}$ and Extended Data Fig. 3a-c). Human THP-1 monocytes showed inflammasome activation upon TcdB stimulation whereas U937 cells did not (Extended Data Fig. 3d-f). Consistently, Pyrin expression in U937 cells was six times lower than that in THP-1 cells (Extended Data Fig. 3g). Ectopic expression of Pyrin in U937 cells regained TcdB-induced caspase 1 activation (Extended Data Fig. 3d, f). In DC2.4 cells, TcdB but not $\mathrm{TcdB}^{\mathrm{m}}$ converted enhanced green fluorescent protein (eGFP)-Pyrin from a dispersed localization into large cytosolic aggregates (Fig. 2d). eGFPPyrin indeed co-aggregated with endogenous ASC, which did not occur with poly(dA:dT) that triggered ASC aggregation through AIM2 inflammasome activation (Fig. 2d and Extended Data Fig. 3h).

Small interference RNA (siRNA) knockdown of Pyrin in primary BMDMs inhibited TcdB-induced caspase 1 activation (Fig. 3a). The knockdown efficiency of three different siRNAs correlated with their inhibition on caspase 1 activation (Fig. 3a, b). We generated Pyrin-deficient mice using transcription activator-like effector nuclease (TALEN)-based genome editing technology and analysed five independent homozygous mutant mice $\left(\mathrm{F}_{1}\right)$ (Extended Data Fig. $4 \mathrm{a}, \mathrm{b}$ ). When BMDMs from Mefv $v^{-l-}$ strains $\left(\mathrm{F}_{1}-1\right.$ and $\left.\mathrm{F}_{1}-2\right)$ were stimulated with TcdB, Salmonella typhimurium flagellin (FliC), or LPS plus nigericin, only TcdBinduced inflammasome activation was diminished (Fig. $3 \mathrm{c}-\mathrm{e}$ ). The results were confirmed in BMDMs from two additional Mefv ${ }^{-1-}$ strains $\left(\mathrm{F}_{1}-3\right.$ and $\left.\mathrm{F}_{1}-4\right)$ (Extended Data Fig. $\left.4 \mathrm{c}\right)$. Thus, Pyrin is required for TcdBinduced inflammasome activation.

Both TcdB and TcsL caused cell rounding owing to an inactivating modification of Rho GTPases (Extended Data Figs 2a and 5a-c), suggesting that actin cytoskeleton disruption is unlikely to be the cause of Pyrin activation. Supporting this idea, cytochalasin D and the actin crosslinking domain (ACD) from Vibrio cholerae RTX toxin ${ }^{14}$ induced severe cell rounding but no evident caspase 1 activation (Extended Data Fig. 6a, b). The RID domain of Vibrio RTX toxin that induces Rho-GTP hydrolysis without covalent modification ${ }^{15}$ did not stimulate inflammasome activation in primary BMDMs and Pyrin-reconstituted DC2.4 and 293T cells (Extended Data Fig. 6c-e). Thus, Pyrin specifically responds to toxin-catalysed inactivating modifications of Rho GTPases.

Rho GTPases are frequent targets of bacterial pathogens ${ }^{16}$. Recent studies identify several FIC-domain Rho-adenylylating effectors: $V$. parahaemolyticus VopS adenylylates the same threonine as $\mathrm{TcdB}^{17}$ (Thr 37 in RHOA; Thr 35 in Rac/Cdc42) and the two FIC domains in H. somni IbpA modify a nearby tyrosine (Tyr 34 in RHOA; Tyr 32 in Rac/ Cdc42 $)^{18,19}$. Similar to TcdB, the three FIC domains target the Rho subfamily and Rac/Cdc42 but not other Ras-superfamily members ${ }^{18}$. When purified VopS or IbpA-Fic1/2 was delivered into primary BMDMs using an anthrax lethal factor N-terminal domain (LFn)-fusion strategy, apparent cell rounding and covalent modification of RHOA occurred (Extended Data Figs $2 \mathrm{~b}$ and $5 \mathrm{~d}$ ), both of which were abolished by mutation of the catalytic histidine (H348A in VopS, H3295A in IbpA-Ficl and H3717A in IbpA-Fic2). All three FIC-domain proteins, but not their catalytic histidine mutants, induced evident caspase 1 inflammasome activation, which required $A s c$ but not Nlrp3 and Nlrc4 (Extended Data Fig. 7a, b). In addition, similar to TcdB, VopS and IbpA-Fic1/2-induced inflammasome activation was diminished in $M e f v^{-1-}$ BMDMs (Fig. 4a and Extended Data Fig. 7c, d). VopS-positive V. parahaemolyticus induced robust caspase 1 activation in Pyrin-reconstituted DC2.4 cells but a weak Pyrin-independent response in primary BMDMs (Extended Data Fig. 7e, f). Biochemical activation of the Pyrin inflammasome observed with recombinant VopS and IbpA-Fic1/2 further supports that Pyrin senses pathogen modification of Rho GTPases.

TcdB and FIC-domain effectors modify both the Rho subfamily (RHOA/B/C) and Rac/Cdc42, whereas TcsL only targets Rac/Cdc42, Ras and Ras-related Ral/Rap GTPases ${ }^{20,21}$. We confirmed that RHOA was inactivated in TcdB but not TcsL-stimulated BMDMs whereas cellular Rac and Cdc42 were inactivated by both toxins (Extended Data Fig. 5b, c). C. botulinum C3 toxin, the first and most established Rhomodifying toxin ${ }^{22}$, is highly selective for $\mathrm{RHOA} / \mathrm{B} / \mathrm{C}^{16}$. C3 ADP-ribosylates Asn 41 in RHOA, causing a slower migration on an SDS-PAGE gel (Extended Data Fig. 8a). C3 and TcdB modifications are mutually exclusive owing to the physical proximity between Asn 41 and Thr 37 (in RHOA). Taking advantage of this property, we confirmed that endogenous
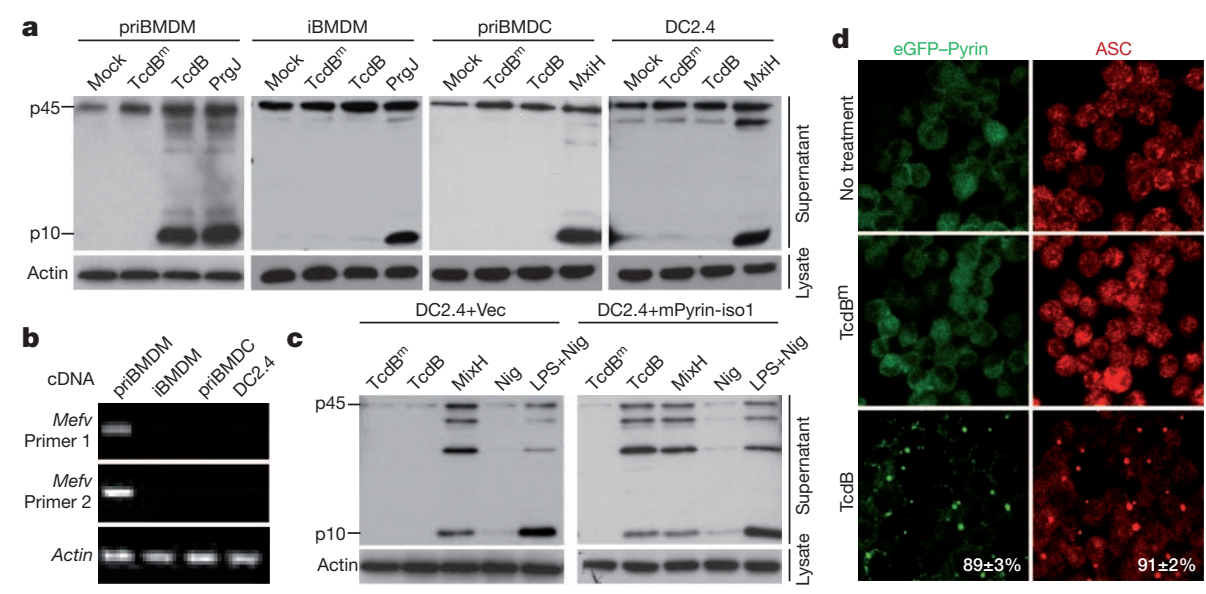

Figure $2 \mid$ Pyrin mediates TcdB-induced inflammasome activation. a, b, Profiling of the sensitivity of different macrophage/dendritic cells to $\mathrm{TcdB}$ and their Pyrin expression. Mouse primary BMDMs (priBMDM), immortalized BMDMs (iBMDM), primary bone marrow-derived dendritic cells (priBMDC) and DC2.4 cells were stimulated as indicated. PrgJ and $\mathrm{MxiH}$ were delivered by the LFn-PA (protective antigen) system. Mefv mRNA levels in $\mathbf{b}$ were measured by reverse transcriptase (RT)-PCR. c, d, TcdBinduced inflammasome activation in Pyrincomplemented DC2.4 cells. DC2.4 cells harbouring a vector $(\mathrm{Vec})$ or mouse Pyrin isoform 1 (mPyriniso1) or stably expressing eGFP-Pyrin were stimulated as indicated (Nig, nigericin). Caspase 1 immunoblots are shown in a and $\mathbf{c}$; eGFP and antiASC fluorescence images are in $\mathbf{d}$. Data in all panels are representative from at least three repetitions. 

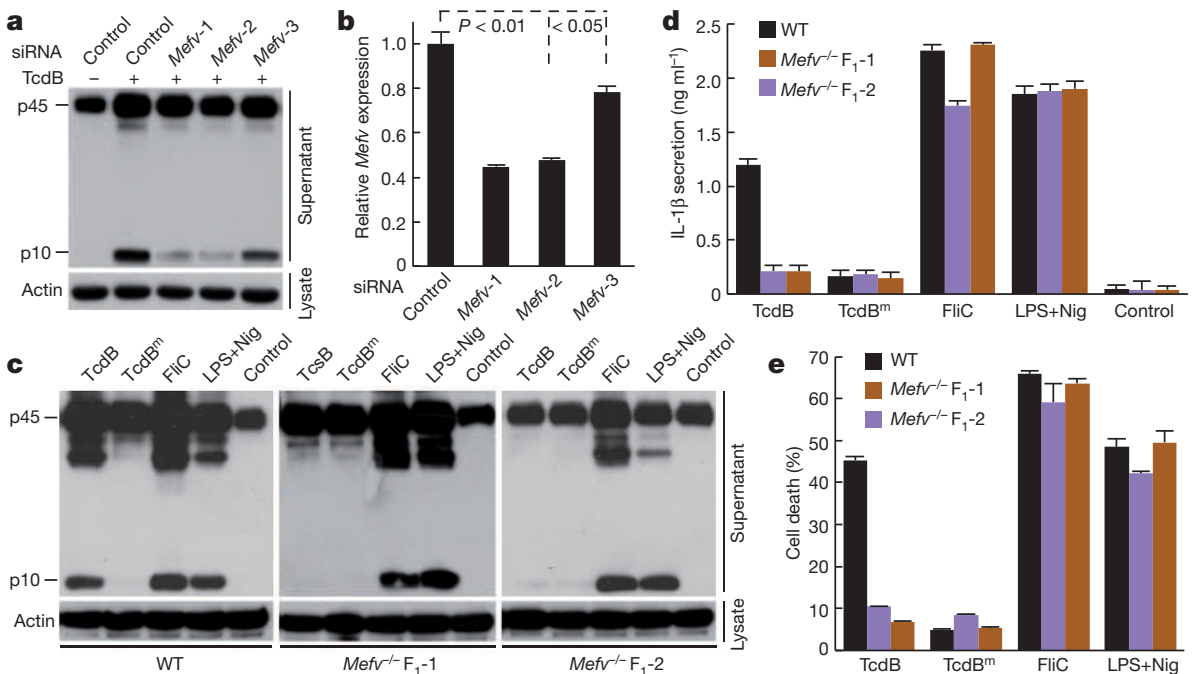

Figure $3 \mid$ Pyrin is required for TcdB-induced inflammasome activation. a, b, Effects of Mefv knockdown on TcdB-induced caspase 1 activation in primary BMDMs. The knockdown efficiency of three independent $M e f v$-targeting siRNAs $(M e f v-1 / 2 / 3)$ was measured by quantitative (q)RT$\mathrm{PCR}$ in $\mathbf{b}(n=3$, mean \pm s.d.; $P$ value, Student's $t$-test). c-e, Effects of Mefv knockout on TcdBinduced inflammasome activation. Mefv ${ }^{-1-}$ mice were generated by TALEN-mediated targeting (Extended Data Fig. 4). FliC (S. typhimurium flagellin) was delivered by the LFn-PA system. $\mathrm{F}_{1}-1$ and $\mathrm{F}_{1}-2$ are two independent $M e f v^{-/-} \mathrm{F}_{1}$ lines. Representative caspase 1 immunoblots from at least three repetitions are in a and $\mathbf{c}$; ELISA assays of IL- $1 \beta$ release and cell death measured by LDH release are in $\mathbf{d}$ and e, respectively $(n=3$; mean \pm s.d.).
RHOA in BMDMs was modified by TcdB but not TcsL (Extended Data Fig. 5a) whereas C3 toxin modified RHOA but not Cdc42 (Extended Data Fig. 8b). Consistently, RHOA but not Rac/Cdc42 was inactivated in C3-intoxicated macrophages, contrary to TcsL stimulation (Extended Data Fig. 5b, c). Similar to TcdB, C3 triggered extensive caspase 1 activation and IL- $\beta$ secretion in primary BMDMs (Extended Data Fig. $8 c, d$ ). The catalytically inactive $\mathrm{C} 3^{\mathrm{m}}$ mutant (Q212A/E214A) that did not modify cellular RHOA (Extended Data Fig. 8a) induced no inflammasome activation (Extended Data Fig. 8c, d). C3-induced inflammasome activation was independent of NLRP3 and NLRC4, but abolished in $A s \mathrm{C}^{-1-}$ and $M e f v^{-1-}$ BMDMs (Fig. $4 \mathrm{~b}$ and Extended Data Fig. 8c-e). A TcsL H17-C variant (replacing $\alpha 17$ in TcsL with the corresponding helix from TcdB) could recognize RHOA and catalyse the same modification as $\mathrm{TcdB}^{23}$ (Fig. 4c). TcsL H17-Calso induced caspase 1 activation, which was diminished in $M e f v^{-1-}$ BMDMs (Fig. 4d). The modification sites by TcdB, FIC-domain effectors and C3 toxin (Thr 37, Tyr 34 and Asn 41 in RHOA) are all in the GTPase switch-I region. These together strongly suggest that switch-I modification of RHOA or other Rho-subfamily members but not Rac/Cdc42 induces Pyrin inflammasome activation.

In a 293T cell reconstitution system (stably expressing Pyrin and RFP-ASC), when expression of RHOA/B/C was individually knocked down by specific siRNAs (Extended Data Fig. 9a), percentages of cells developing RFP-ASC foci upon TcdB stimulation were not significantly decreased (Extended Data Fig. 9b). However, triple knockdown of RHOA/B/C markedly reduced TcdB-induced RFP-ASC foci formation (Fig. 4e and Extended Data Fig. 9b). Similar results were obtained with another independent set of siRNAs (Extended Data Fig. 9c, d). The deficient ASC foci formation in the triple-knockdown cells could be restored by stable expression of RNAi-resistant RHOA whereas the modification-site mutant of RHOA(T37A) showed no rescue effects (Fig. 4f). These results establish the requirement of Rho modification for toxin-induced Pyrin activation and also indicate a functional redundancy of RHOA/B/C. In both $293 \mathrm{~T}$ and DC2.4 cells, no PyrinRho interaction could be detected even in the presence of TcdB (Extended
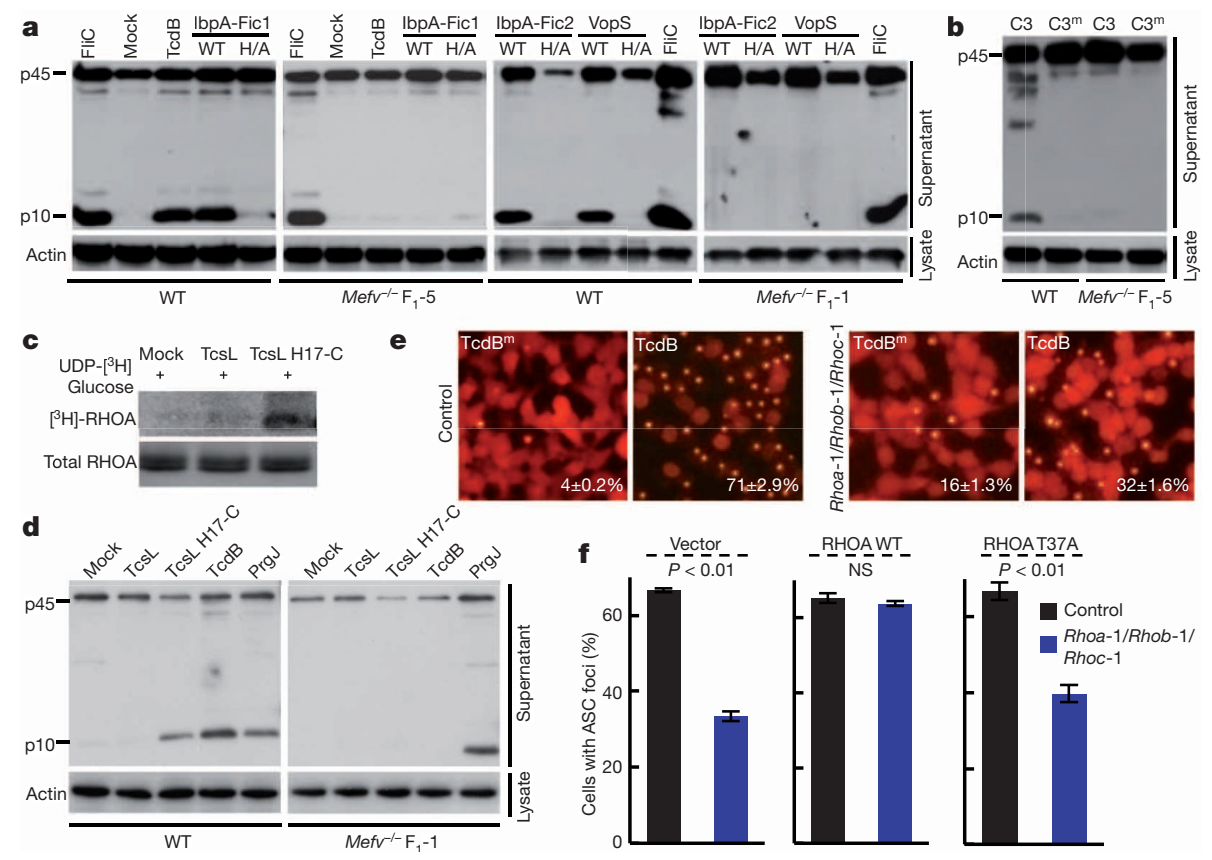

Figure $4 \mid$ Switch-I modification of the Rho subfamily accounts for Pyrin activation by TcdB and other Rho-modifying toxins. $\mathbf{a}, \mathbf{b}$, Caspase 1 activation by $V$. parahaemolyticus VopS, H. somni IbpA (IbpA-Fic1/2) and C. botulinum C3 toxin (delivered into BMDMs by the LFn-PA system) and effects of Mefv knockout. H/A, mutation of the FIC-domain catalytic histidine; $\mathrm{C}^{\mathrm{m}}$, the catalytically inactive Q212A/E214A mutant. c, In vitro glucosylation of RHOA by TcsL and TcsL H17-C. Shown are ${ }^{3} \mathrm{H}$ autoradiograph and immunoblot of RHOA. d, Wild-type or Mefv ${ }^{-1-}$ BMDMs were treated with TcsL H17-C or indicated control stimuli. $\mathbf{e}, \mathbf{f}$, Requirement of the Rho subfamily and its modification for TcdBinduced inflammasome activation. 293T cells stably expressing Pyrin and RFP-ASC were transfected with control or a pool of three siRNAs targeting RHOA, B and C, respectively (Rhoa-1, Rhob-1 and Rhoc-1). The cells were additionally stable-transfected with a vector (left), or RNAiresistant RHOA wild type (WT; middle) or T37A (right) before Rho knockdown and TcdB stimulation in $\mathbf{f}$. Percentages of cells showing the ASC foci (marked on the fluorescence images in $\mathbf{e}$ ) are mean \pm s.d. $(n=3)$ ( $P$ value, Student's $t$-test; NS, not significant). Representative caspase 1 immunoblots from at least three repetitions are shown in $\mathbf{a}, \mathbf{b}$ and $\mathbf{d}$. 
Data Fig. 9e, f), suggesting a different mechanism of Pyrin sensing of Rho modification/inactivation from the NOD2-RIP2 axis that directly binds to Rho-GTP ${ }^{12,24,25}$.

Intracellular B. cenocepacia causes fatal chronic lung infection in immunocompromised individuals. B. cenocepacia inactivates Rho and disrupts actin cytoskeleton in a T6SS-dependent manner ${ }^{26,27}$, which was confirmed in DC2.4 cells (Extended Data Fig. 5e). RHOA in wild-type B. cenocepacia-infected but not T6SS-deficent $\Delta h c p$ strain-infected macrophages was resistant to $\mathrm{C} 3$ modification, suggesting a modification by B. cenocepacia (Fig. 5a). Mass spectrometric analysis of Flag-RHOA purified from uninfected or B. cenocepacia-infected DC2.4 cells was therefore performed. Among all identified tryptic peptides covering $97 \%$ of the RHOA sequence, modification of one peptide, ${ }_{28} \mathrm{DQFPE}$ VYVPTVFENYVADIEVDGK ${ }_{51}$, was found in B. cenocepacia-infected cells; tandem mass spectrometry revealed that Asn 41 within the peptide was deamidated into an aspartate (Fig. 5b). The extracted ion chromatogram showed that more than $90 \%$ of Asn 41 -containing peptides recovered from wild-type infection were deamidated whereas Asn 41-deamidated peptide was barely detectable in uninfected or $\Delta h c p$ infected cells (Fig. 5c). Thus, B. cenocepacia infection induces RHOA deamidation on Asn 41 in a T6SS-dependent manner.

Asn 41 is the same site modified by $\mathrm{C} 3$ toxin, indicating a role of Pyrin in defending B. cenocepacia infection. A recent study reports that Pyrin knockdown decreases IL- $1 \beta$ secretion in B. cenocepacia-infected human monocytes ${ }^{28}$. We observed efficient caspase 1 processing in wildtype B. cenocepacia but not $\Delta h c p$-infected mouse BMDMs (Extended Data Fig. 10a). Similarly to that observed with TcdB, Nlrp3 ${ }^{-/-} \mathrm{Nlrc4} 4^{-1-}$ and Aim $2^{-1-}$ BMDMs showed intact inflammasome response to B. cenocepacia (Extended Data Fig. 10b, c), whereas little caspase 1 activation and IL- $1 \beta$ secretion were detected in infected $\mathrm{Mefv}^{-/-}$and $\mathrm{Asc}^{-/-}$ BMDMs (Fig. 5d and Extended Data Fig. 10b-e). Mefv ${ }^{-1-}$ did not affect S. typhimurium-induced inflammasome activation. Furthering these genetic analyses, re-expression of Pyrin in DC2.4 and U937 cells restored caspase 1 inflammasome activation by $B$. cenocepacia (but not EHEC, which harbours no Rho-modifying effectors), which remained dependent upon the T6SS (Extended Data Fig. 10f-i). In the 293T cell

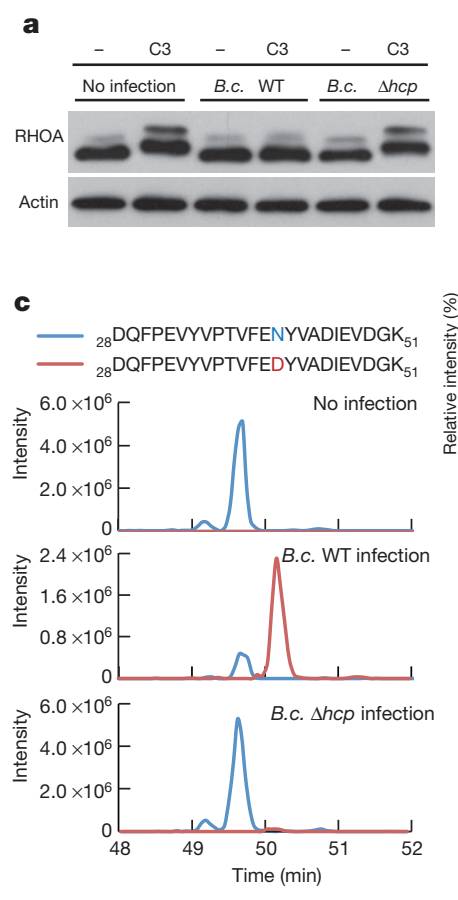

e

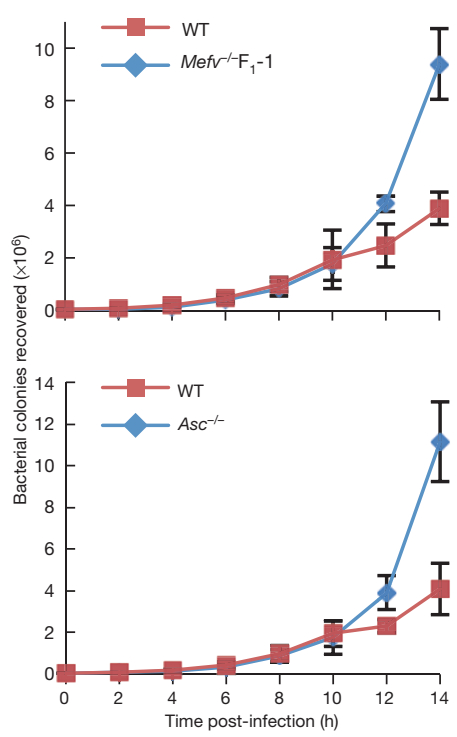

e

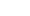

b

.

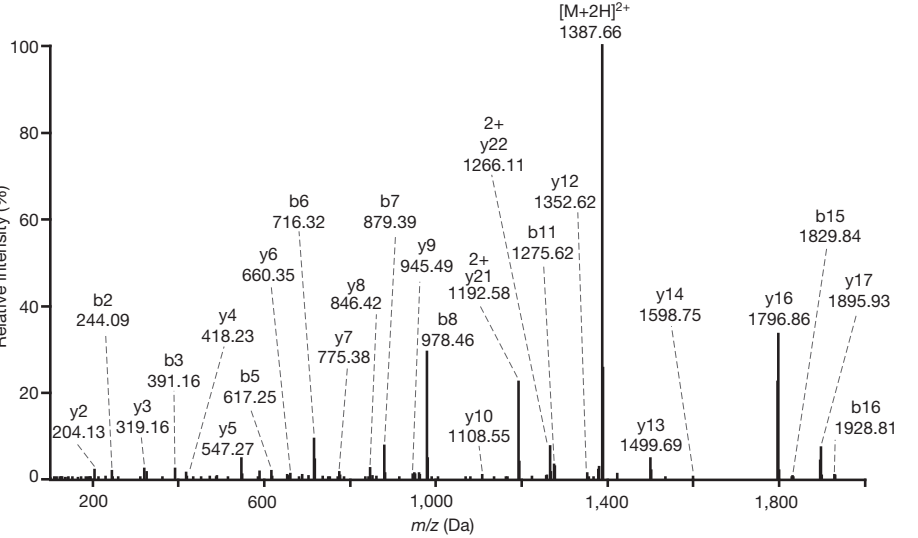

d
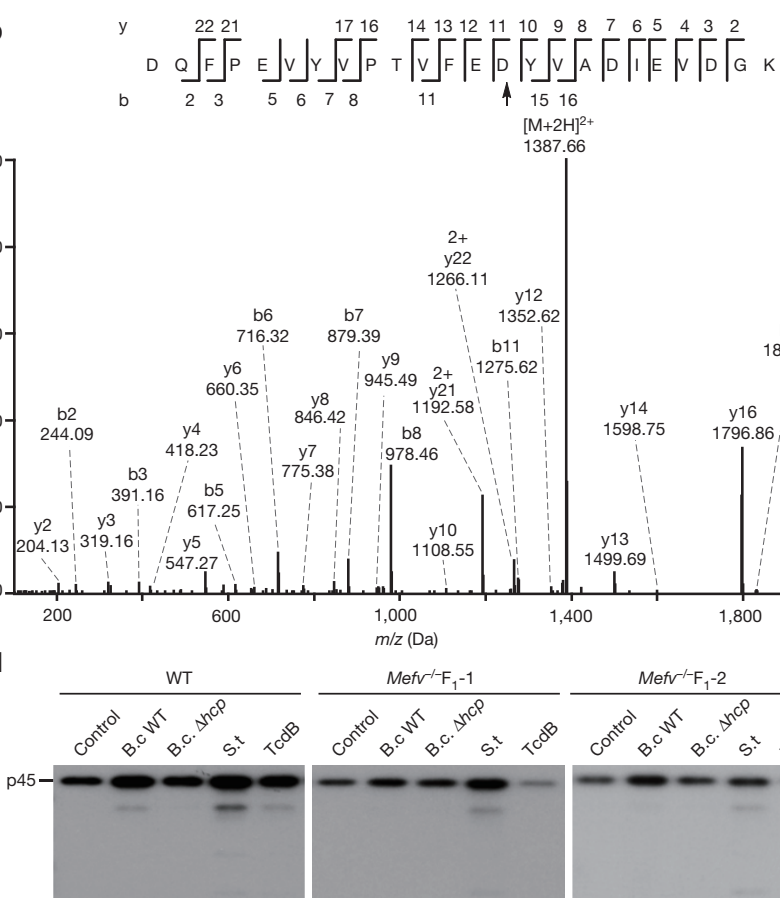$$
1387
$$
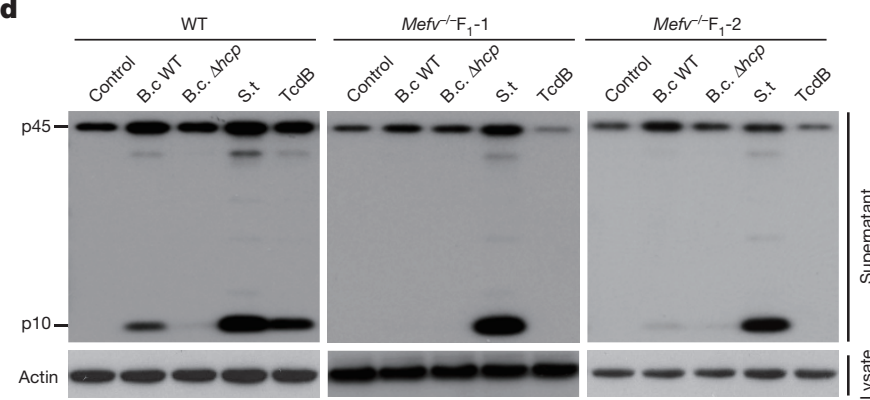

f

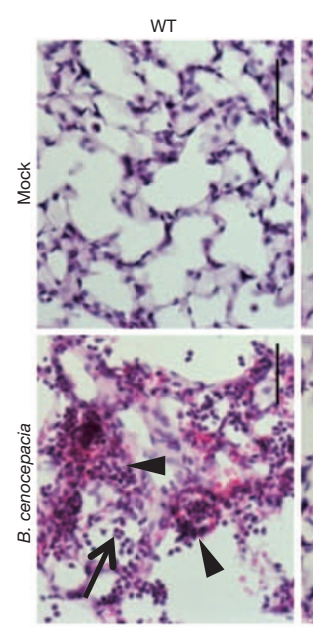

Figure 5 T6SS-dependent Asn 41 deamidation of RHOA by B. cenocepacia activates the Pyrin inflammasome. a, B. cenocepacia infection-induced RHOA modification in BMDMs probed by the sensitivity to in vitro $\mathrm{C} 3$ modification. $\mathbf{b}$, Tandem mass spectrum of a tryptic peptide of Flag-RHOA from wild-type B. cenocepacia-infected DC2.4 cells. The b- and y-type product ions are marked and illustrated along the peptide sequence shown on top of the spectrum. Arrowhead, the deamidated Asn 41. c, Flag-RHOA purified from uninfected or B. cenocepacia-infected DC2.4 cells was analysed by mass spectrometry. Shown are extracted ion chromatograms of the Asn 41containing peptide. d, Effects of Mefv knockout on B. cenocepacia-induced caspase 1 activation in primary BMDMs. e, Effects of Mefv and Asc knockout on B. cenocepacia intracellular replication. The numbers of bacterial colonies recovered from BMDM lysates $(n=3$; mean \pm s.d.) are plotted against the time duration of infection. f, Indicated mice were infected with B. cenocepacia or a buffer control (mock).

Representative haematoxylin \& eosin staining of the lung sections is shown. Black triangle and arrow mark inflammatory cell infiltration and intra-alveolar leukocytes, respectively. Scale bars, $50 \mu \mathrm{m}$. B. c. WT and $\Delta h c p$ indicate wild-type and T6SS-deficient B. cenocepacia J2315, respectively. Data in all panels are representative of at least three repetitions. 
reconstitution system, overexpression of deamidated (N41D) but not wild-type RHOA could drive RFP-ASC foci formation when endogenous RHOA/B/C were simultaneously knocked down (Extended Data Fig. 10j). This confirms that B. cenocepacia-induced RHOA deamidation accounts for Pyrin inflammasome activation. Furthermore, B. cenocepacia replication in $\mathrm{Mefv}^{-1-}$ and $\mathrm{Asc}^{-/-}$BMDMs was comparable but much higher than that in wild-type BMDMs (Fig. 5e). B. cenocepacia-infected mice developed strong lung inflammation, marked by inflammatory cell infiltration, appearance of intra-alveolar leukocytes and disruption of the normal lung architecture. These responses were severely attenuated in the lungs of infected $M e f v^{-1-}$ and $A s c^{-1-}$ mice (Fig. 5f). Thus, the Pyrin inflammasome is a critical for immune defence against $B$. cenocepacia by sensing bacterial T6SSinduced deamidation of Rho GTPase.

Here we discover that the FMF disease protein Pyrin is a specific immune sensor for bacterial modifications of Rho GTPases. Common to all identified bacterial stimuli is the modification of a switch-I residue in Rho-subfamily and GTPase inactivation. The modifications cover glucosylation, adenylylation, ADP-ribosylation and deamidation occurring on different residues. Thus, Pyrin does not directly recognize Rho modification, but probably senses an event downstream of Rho modification in the actin cytoskeleton pathway. Interestingly, direction interaction of Pyrin with actin and co-localization of Pyrin-ASC complex with actin filaments are observed ${ }^{29}$. Pyrin detects pathogen virulence activity, which is different from most mammalian PRRs that directly recognize microbial products. The disease resistance PRR protein in plant innate immunity often acts in an indirect manner by detecting pathogen-induced modification of a host protein, a model known as guard hypothesis ${ }^{30}$. Thus, the mode of Pyrin action echoes the plant guard model to some extent.

\section{METHODS SUMMARY}

Purified recombinant TcdB or TcsL was added into serum-free macrophage culture medium at a final concentration of $0.1 \mu \mathrm{g} \mathrm{ml}^{-1}$ or that indicated for $2.5-3 \mathrm{~h}$. Supernatants of BMDMs $\left(5 \times 10^{5}\right)$ or DC2.4 cells $\left(1 \times 10^{6}\right)$ were subjected to trichloroacetic acid precipitation and the precipitates were analysed by anti-caspase 1 immunoblotting to detect pro-caspase 1 (p45) and the processed p10 fragment; cell lysates were blotted with anti-Actin antibody to ensure equal loading. To measure IL-1 $\beta$ secretion, BMDMs or DC2.4 cells were primed with LPS $\left(500 \mathrm{ng} \mathrm{ml}^{-1}, 2 \mathrm{~h}\right)$, and released mature IL-1 $\beta$ was determined using the IL-1 $\beta$ ELISA kit (Neobioscience Technology Company). Cell pyroptosis was measured by the lactate dehydrogenase assay using CytoTox 96 Non-Radioactive Cytotoxicity Assay kit (Promega). All independent experiments carried out in this study and indicated in the figure legends were biological replicates.

Online Content Methods, along with any additional Extended Data display items and Source Data, are available in the online version of the paper; references unique to these sections appear only in the online paper.

Received 6 October 2013; accepted 7 May 2014.

Published online 11 June 2014.

1. Seshadri, S., Duncan, M. D., Hart, J. M., Gavrilin, M. A. \& Wewers, M. D. Pyrin levels in human monocytes and monocyte-derived macrophages regulate IL-1 $\beta$ processing and release. J. Immunol. 179, 1274-1281 (2007).

2. Yu, J. W. et al. Cryopyrin and pyrin activate caspase-1, but not NF-kB, via ASC oligomerization. Cell Death Differ. 13, 236-249 (2006).

3. French FMF Consortium. A candidate gene for familial Mediterranean fever. Nature Genet. 17, 25-31 (1997).

4. The International FMF Consortium. Ancient missense mutations in a new member of the RoRet gene family are likely to cause familial Mediterranean fever. Cell 90, 797-807 (1997).

5. Chae, J. J. et al. Gain-of-function Pyrin mutations induce NLRP3 proteinindependent interleukin-1 $\beta$ activation and severe autoinflammation in mice. Immunity 34, 755-768 (2011).

6. Jank, T. \& Aktories, K. Structure and mode of action of clostridial glucosylating toxins: the ABCD model. Trends Microbiol. 16, 222-229 (2008).
7. Kuehne, S. A. et al. The role of toxin $A$ and toxin $B$ in Clostridium difficile infection. Nature 467, 711-713 (2010).

8. Lyras, D. et al. Toxin B is essential for virulence of Clostridium difficile. Nature 458, 1176-1179 (2009).

9. Kayagaki, N. et al. Non-canonical inflammasome activation targets caspase-11. Nature 479, 117-121 (2011)

10. Ng, J. et al. Clostridium difficile toxin-induced inflammation and intestinal injury are mediated by the inflammasome. Gastroenterology 139, 542-552.e1-3 (2010).

11. Keestra, A. M. et al. Manipulation of small Rho GTPases is a pathogen-induced process detected by NOD1. Nature 496, 233-237 (2013).

12. Fukazawa, A. et al. GEF-H1 mediated control of NOD1 dependent NF-kB activation by Shigella effectors. PLoS Pathog. 4, e1000228 (2008).

13. Richards, N. et al. Interaction between pyrin and the apoptotic speck protein (ASC) modulates ASC-induced apoptosis. J. Biol. Chem. 276, 39320-39329 (2001).

14. Cordero, C. L., Kudryashov, D. S., Reisler, E. \& Satchell, K. J. The actin cross-linking domain of the Vibrio cholerae RTX toxin directly catalyzes the covalent crosslinking of actin. J. Biol. Chem. 281, 32366-32374 (2006)

15. Sheahan, K. L. \& Satchell, K. J. Inactivation of small Rho GTPases by the multifunctional RTX toxin from Vibrio cholerae. Cell. Microbiol. 9, 1324-1335 (2007).

16. Aktories, K. Bacterial protein toxins that modify host regulatory GTPases. Nature Rev. Microbiol. 9, 487-498 (2011)

17. Yarbrough, M. L. et al. AMPylation of Rho GTPases by Vibrio VopS disrupts effector binding and downstream signaling. Science 323, 269-272 (2009).

18. Mattoo, S. et al. Comparative analysis of Histophilus somni immunoglobulinbinding protein $\mathrm{A}(\mathrm{IbpA})$ with other fic domain-containing enzymes reveals differences in substrate and nucleotide specificities. J. Biol. Chem. 286, 32834-32842 (2011).

19. Worby, C. A. et al. The fic domain: regulation of cell signaling by adenylylation. Mol. Cell 34, 93-103 (2009)

20. Popoff, M. R. et al. Ras, Rap, and Rac small GTP-binding proteins are targets for Clostridium sordellii lethal toxin glucosylation. J. Biol. Chem. 271, 10217-10224 (1996).

21. Just, I., Selzer, J., Hofmann, F., Green, G. A. \& Aktories, K. Inactivation of Ras by Clostridium sordellii lethal toxin-catalyzed glucosylation. J. Biol. Chem. 271, 10149-10153 (1996).

22. Aktories, K. \& Hall, A. Botulinum ADP-ribosyltransferase $C_{3}$ : a new tool to study low molecular weight GTP-binding proteins. Trends Pharmacol. Sci. 10, 415-418 (1989).

23. Jank, T., Giesemann, T. \& Aktories, K. Clostridium difficile glucosyltransferase toxin B-essential amino acids for substrate binding. J. Biol. Chem. 282, 35222-35231 (2007).

24. Boyer, L. et al. Pathogen-derived effectors trigger protective immunity via activation of the Rac2 enzyme and the IMD or Rip kinase signaling pathway. Immunity 35, 536-549 (2011)

25. Bruno, V. M. et al. Salmonella Typhimurium type III secretion effectors stimulate innate immune responses in cultured epithelial cells. PLoS Pathog. 5, e1000538 (2009).

26. Flannagan, R. S. etal. Burkholderia cenocepacia disrupts host cell actin cytoskeleton by inactivating Rac and Cdc42. Cell. Microbiol. 14, 239-254 (2012).

27. Rosales-Reyes, R., Skeldon, A. M., Aubert, D. F. \& Valvano, M. A. The Type VI secretion system of Burkholderia cenocepacia affects multiple Rho family GTPases disrupting the actin cytoskeleton and the assembly of NADPH oxidase complex in macrophages Cell Microbiol 14, 255-273 (2012)

28. Gavrilin, M. A. et al. Activation of the pyrin inflammasome by intracellular Burkholderia cenocepacia. J. Immunol. 188, 3469-3477 (2012).

29. Waite, A. L. et al. Pyrin and ASC co-localize to cellular sites that are rich in polymerizing actin. Exp. Biol. Med. (Maywood) 234, 40-52 (2009).

30. Jones, J. D. \& Dangl, J. L. The plant immune system. Nature 444, 323-329 (2006)

Acknowledgements We thank V. Dixit for providing knockout mice and anti-ASC antibody, D. Lyras for C. sordellii genomic DNA, J. Xiao for IbpA-Fic constructs, T. lida for V. parahaemolyticus strain, M. Valvano for pDAl-Scel vector and $H$. Feng for TcdB B. megaterium expression system. We thank members of the Shao laboratory for discussions. The research was supported in part by an International Early Career Scientist grant from the Howard Hughes Medical Institute to F.S. This work was also supported by the National Basic Research Program of China 973 Program (2012CB518700), the Strategic Priority Research Program of the Chinese Academy of Sciences (XDB08020202) and China National Science Foundation Program for Distinguished Young Scholars (31225002) to F.S

Author Contributions F.S. conceived the study; H.X. and J.Y. performed the majority of experiments, assisted by W.G.; L.L., P.L., L.Z., Y.-N.G., X.P., J.J.X., S.C. and F.W. contributed reagents and analytic tools. H.X., J.Y. and F.S. analysed the data and wrote the manuscript. All authors discussed the results and commented on the manuscript.

Author Information Reprints and permissions information is available at www.nature.com/reprints. The authors declare no competing financial interests. Readers are welcome to comment on the online version of the paper. Correspondence and requests for materials should be addressed to F.S. (shaofeng@nibs.ac.cn). 


\section{METHODS}

Plasmids, antibodies and reagents. Complementary DNAs for mouse Pyrin isoform 1 (NM_001161790.1) and isoform 2 (NM_001161791.1) were amplified from reverse-transcribed cDNA from BMDMs. cDNA for human Pyrin isoform 1 (NM 000243.2) was synthesized by the in-house facility. Human ASC and NLRP3 cDNA were described previously ${ }^{31}$ and mouse NLRP1B cDNA was kindly provided by R. Vance (University of California, Berkeley). cDNAs for $h N L R P 2, h N L R P 5, h N L R P 7$, $h N L R P 8, h N L R P 9, h N L R P 11$ and $h N L R P 12$ were amplified from IMAGE clones (2821428, 100014773, 40036028, 100016540, 9020573, 4341197 and 5212737, respectively). $h N L R P 6$ cDNA (FLJ77198) was obtained from Biological Resource Center (NBRC), National Institute of Technology and Evaluation (NITE), Japan. All above genes were inserted into pCS2-6 $\times$ Myc or pWPI-IRES-eGFP vectors for expression in 293T and DC2.4 cells. pHIS1522-TcdB for expression in Bacillus megaterium was kindly provided by H. Feng (University of Maryland, Baltimore). DNA for TcsL, amplified from C. sordellii genome DNA, was digested with BsrG I/KpnI and inserted into the pHIS1522 vector. DNA for C. botulinum C3 toxin was provided by A. Hall (Memorial Sloan-Kettering Cancer Center, New York). VopS was amplified from $V$. parahaemolyticus genomic DNA. cDNAs for H. somni IbpA-Ficl and Fic2 domains were provided by J. Xiao (University of California, San Diego). cDNA encoding the ACD and RID domains of RTX toxin was amplified from $V$. cholera El Tor N16961 strain genomic DNA. All the bacterial effectors were cloned into the pET28a-LFn vector for expression in E. coli. Point mutations were generated by the QuickChange Site-Directed Mutagenesis Kit (Stratagene). All plasmids were verified by DNA sequencing.

Antibodies for caspase 1 (sc-515), RHOA (sc-428), Cdc42 (sc-8401) and Racl (sc-95) were obtained from Santa Cruz Biotechnology. Anti-ASC antibody was provided by V. Dixit (Genentech). Anti-Actin antibody (A2066) and cytochalasin D (C8273) were purchased from Sigma. Alexa Fluor 546 goat anti-rat IgG secondary antibody (A-11081) was from Invitrogen. Cell culture products were from Invitrogen and all other chemicals were Sigma-Aldrich products unless noted.

Cell culture, bone marrow macrophages and transfection. 293T cells and an immortalized macrophage line derived from C57BL/6 mice were grown in DMEM

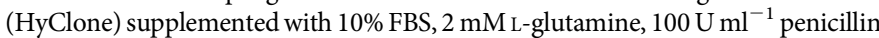
and $100 \mathrm{mg} \mathrm{ml}^{-1}$ streptomycin. Human U937 and THP-1 monocytes from ATCC and mouse DC2.4 dendritic cell line were cultured in RPMI-1640 medium containing 10\% FBS and $2 \mathrm{mM}$ L-glutamine. U937 and THP-1 cells were differentiated into macrophages by PMA as previously described ${ }^{32}$.

Wild-type C57BL/6 mice and all other inbred strains (DBA/2, C3H/He, KM, 129 S and ICR) were from Vital River Laboratory Animal Technology Co. Caspase $1^{-/-}$mice were obtained from the Jackson Laboratory. Nlrc4 ${ }^{-1-}, \mathrm{Nlrp}^{-/-}, \mathrm{Aim}^{-/-}$ and $A s c^{-l-}$ mice were provided by V. Dixit (Genentech), and used to generate the $N l r c 4^{-1-} \mathrm{Nlrp} 3^{-1-}$ double-knockout strain. All knockout alleles have been crossed onto the C57BL/6 background. Animal experiments were conducted following the Ministry of Health national guidelines for housing and care of laboratory animals and performed in accordance with institutional regulations after review and approval by the Institutional Animal Care and Use Committee at National Institute of Biological Sciences. Primary BMDMs were prepared by following a standard protocol described previously ${ }^{32}$. All cells were cultivated in a humidified atmosphere containing $5 \% \mathrm{CO}_{2}$ at $37^{\circ} \mathrm{C}$. jetPRIME (Polyplus Transfection) was used for 293T cell transfection by following the manufacturer's instructions.

Stable cell line and co-immunoprecipitation. For lentivirus packaging, $1.0 \mu \mathrm{g}$ pWPI-IRES-eGFP plasmid harbouring the indicated inserts, $0.35 \mu \mathrm{g} \mathrm{pMD} 2 . \mathrm{G}$ and $0.7 \mu \mathrm{g}$ psPAX2 plasmids (Addgene) were co-transfected into $293 \mathrm{~T}$ cells in the sixwell plate $\left(5 \times 10^{5}\right.$ per each well). $24 \mathrm{~h}$ post transfection, the medium was changed into $3 \mathrm{ml}$ HEPES-buffered Opti-MEM (Invitrogen) supplemented with 20\% FBS and $2 \mathrm{mM}$ L-glutamine. $36-48 \mathrm{~h}$ post transfection, supernatants containing lentivirus particles were collected and $1.5 \mathrm{ml}$ of the supernatants were used to infect DC2.4, 293T or U937 cells. $72 \mathrm{~h}$ post infection, infected cells were isolated by fluorescenceactivated cell sorting on the FACSAria II flow cytometer (BD Biosciences). For stable expression of eGFP-Pyrin and RFP-ASC in 293T cells, the cells were sorted into single clones to obtain stable cell lines with the lowest background of ASC aggregation. To examine the possible interaction between Pyrin and Rho GTPases, antiFlag co-immunoprecipitation was performed in transiently transfected 293T cells as well as Pyrin-stable expression DC2.4 cells following the procedure described previously $^{32}$

ASC foci formation. Indicated BMDM, DC2.4 or $293 \mathrm{~T}$ cells cultured in the 24well plate were stimulated with $\mathrm{TcdB}$ or other indicated toxins. Cells were washed three times with pre-cooled PBS and fixed by $4 \%$ paraformaldehyde. Cells were then permeabilized with $0.1 \%$ Triton X-100 in PBS for 10 min. RFP-ASC was imaged directly and endogenous ASC was stained by anti-ASC antibody and Alexa Fluor 546 goat anti-rat IgG secondary antibody. ASC foci formation was detected by the Olympus IX71 microscope. The captured pictures were processed by the Image] program. For each picture, a total of 200-300 cells from three randomly selected fields were counted and statistical data were analysed to give the average ratio and standard error.

siRNA knockdown. To knockdown Mefv expression, $1 \mu \mathrm{l}$ control or Mefv-specific siRNAs (final concentration, $50 \mathrm{nM}$ ) were transfected into $2.5 \times 10^{5}$ BMDMs in the 24 -well plate using $2 \mu \mathrm{l}$ of INTERFERin reagent (Polyplus Transfection). $800 \mu \mathrm{l}$ of pre-warmed fresh RPMI-1640 medium was then added into each well $12 \mathrm{~h}$ after transfection. $36 \mathrm{~h}$ later, BMDMs were treated with indicated stimuli and subjected to caspase 1 inflammasome activation assays. Sequences of the three Mefv-targeting siRNAs used are listed below. Mefv-1, GATGAGATGATTGAAGAACTA; $M e f v$ 2, CCACTTGAGAAGCTATACCAA; Mefv -3, GATCCATTCTCGGAACAAC AA.siRNA knockdown of Nod2 expression in $\mathrm{Nod1}^{-/-}$BMDMs was performed similarly to that described above and the target sequences were GCTCAGGTTG ACTCTGATGAT (Nod2-1) and GCCAACAGTCATACCTTTGAA (Nod2-2). To knockdown human RHOA, B and C expression for ASC foci examination, $1 \times 10^{5}$ 293T cells stably expressing eGEP-Pyrin (mouse Pyrin isoform 2) and RFP-ASC were plated into each well of the 24 -well plate $12 \mathrm{~h}$ before siRNA transfection. 20 pmol total siRNA was used for each transfection at a final concentration of $50 \mathrm{nM}$. For individual knockdown, $6.6 \mathrm{pmol}$ Rho-specific siRNA plus $13.2 \mathrm{pmol}$ control siRNA were co-transfected. To knockdown RHOA, B and C simultaneously, $6.6 \mathrm{pmol}$ of each siRNA was mixed and co-transfected using $2 \mu \mathrm{l}$ of INTERFERin reagent. $500 \mu \mathrm{l}$ of pre-warmed fresh DMEM medium was added into each well $12 \mathrm{~h}$ after transfection. $48 \mathrm{~h}$ post transfection, cells were re-plated into new 24 -well plates at a split ratio of 1:3, followed by a second round of transfection with the same amounts of siRNAs. $36 \mathrm{~h}$ later, cells were treated with $0.5 \mu \mathrm{g} \mathrm{ml}^{-1} \mathrm{TcdB}$ for $5 \mathrm{~h}$ and ASC foci formation was examined. Two sets of siRNA pairs were used and their target sequences are listed below. Rhoa-1, ATGGAAAGCAGGTAGAGTT; Rhoa-2, TGGAAAGACATGCTTGCTCAT; Rhob- 1 , CATCCAAGCCTACGAC TAC; Rhob-2, GCACTTCTGTCCCAATGTG; Rhoc-1, GGATCAGTGCCTTTG GCTA; Rhoc-2, ATAAGAAGGACCTGAGGCA. Knockdown efficiency was determined by $\mathrm{qRT}-\mathrm{PCR}$ analyses.

RT-PCR and qRT-PCR. For RT-PCR analysis, total RNA was extracted by TRIzol (Invitrogen) and digested with DNase I (Invitrogen). $1 \mu \mathrm{g}$ of total RNA was reversetranscribed into cDNA using M-MLV reverse transcriptase (Promega). $0.1 \mu \mathrm{l}$ cDNA was used as the template for a $20-\mu$ reverse-transcription PCR reaction. qRT-PCR analysis was performed using the SYBR Premix Ex Taq (TaKaRa) on Applied Biosystems 7500 Fast Real-Time PCR System; the mRNA level of target genes was normalized to that of Actin for mouse cells or GAPDH for human cells. The primers used for each gene examined are listed below. For human Pyrin, primer 1, TAAG ACCCCTAGTGACCATCTG (forward) and TTCCCCATAGTAGGTGACCAG (reverse); primer 2, GTCGCCCTGGAACACAAGAA (forward) and TCCTCC CCATAGGATCGCTG (reverse).For mouse Pyrin, primer 1, TCATCTGCTAA ACACCCTGGA (forward) and GGGATCTTAGAGTGGCCCTTC (reverse); primer 2, AGGCTTCAAGGACTTTACAACAA (forward) and TCATGCGAATGA GACTCCCA (reverse). For mouse NOD2, GCCCTACAGCTGGATTACAAC (forward) and CGGCTGTGATGTGATTGTTC (reverse).

Purification of recombinant proteins. To purify His-tagged TcdB and TcsL, pHIS1522-TcdB, pHIS1522-TcsL or their mutants were transformed into B. megaterium and recombinant proteins were expressed as previously described ${ }^{33}$.To purify LFn-tagged IbpA-Fic1, IbpA-Fic2, VopS, C3 toxin, MxiH, EprI, PrgJ, the ACD and RID domains of Vibrio RTX toxin, and S. typhimurium FliC proteins, E. coli BL21 (DE3) strains harbouring the corresponding expression plasmids were grown in LB medium supplemented with appropriate antibiotics. Protein expression was induced overnight at $22^{\circ} \mathrm{C}$ with $0.5 \mathrm{mM}$ IPTG when $O D_{600}$ reached 0.8 . Bacteria were harvested and lysed in a buffer containing $50 \mathrm{mM}$ Tris- $\mathrm{HCl}$ (pH 8.0), $300 \mathrm{mM}$ $\mathrm{NaCl}$ and $25 \mathrm{mM}$ imidazole. His-tagged proteins were purified by affinity chromatography using Ni-NTA beads (Qiagen) by following the manufacturer's protocol. Protein antigen (PA)-mediated delivery of LFn-tagged proteins into macrophage or dendritic cells was performed as described previously ${ }^{32,34}$

Rho GTPases activity and modification assays. To examine the mobility shift of RHOA induced by B. cenocepacia $\mathrm{J} 2315$ infection or C3 toxin treatment, lysates of infected or stimulated macrophages were separated on 15\% SDS-PAGE gels, followed by anti-RHOA immunoblotting analysis. To measure the level of Rho-GTP in stimulated macrophage or dendritic cells, GST-RBD (Rho-binding domain of Rhotekin) or PBD (p21-binding domain of PAK1) pulldown assays were performed by following published protocols ${ }^{35,36}$. For C3 ADP-ribosylation of RHOA in the cell extracts, BMDMs were harvested in the buffer $(20 \mathrm{mM} \beta-O G, 0.05 \%$ Triton $\mathrm{X}-100,20 \mathrm{mM}$ Tris- $\mathrm{HCl}, 3 \mathrm{mM} \mathrm{MgCl} 2$ and $1 \mathrm{mM}$ EGTA and a protease inhibitor cocktail, pH 7.5). Sonication was performed to facilitate cell lysis. The lysates were centrifuged at $16,000 \mathrm{~g}$ for $10 \mathrm{~min} .15 \mu \mathrm{l}$ of the supernatants were supplemented with recombinant GST-C3 toxin ( $2 \mu \mathrm{g}$ per each assay), NAD (final concentration, $25 \mu \mathrm{M}$ ) and thymidine (final concentration, $10 \mathrm{mM}$ ). The reaction was performed at $30{ }^{\circ} \mathrm{C}$ for $60 \mathrm{~min}$, stopped by SDS-loading buffer, and then analysed by immunoblotting analysis. To examine Rho modification by TcdB and TcsL directly, 
UDP- $\left[{ }^{3} \mathrm{H}\right]$ Glucose radiolabelling assay was performed with Flag-RHOA/Cdc42 purified from E. coli or DC2.4 stable cells similarly as described previously ${ }^{21}$. Mass spectrometry. DC2.4 cells stably expressing Flag-RHOA were plated at $2 \times$ $10^{6}$ cells per well in a six-well plate. $12 \mathrm{~h}$ later, cells were left untreated or infected with $B$. cenocepacia at an m.o.i. of 20 for $3 \mathrm{~h}$. About $1 \times 10^{8}$ cells for each sample were harvested and lysed in a buffer containing $50 \mathrm{mM}$ Tris- $\mathrm{HCl}$ (pH 7.6), $150 \mathrm{mM}$ $\mathrm{NaCl}$ and $0.5 \%$ Triton X-100 supplemented with a protease inhibitor mixture. Flag-RHOA was purified by anti-Flag affinity chromatograph and eluted with the Flag peptide. Protein bands on the SDS-PAGE gel were de-stained, reduced in $10 \mathrm{mM}$ DTT at $56^{\circ} \mathrm{C}$ for $30 \mathrm{~min}$ followed by alkylation in $55 \mathrm{mM}$ iodoacetamide in dark for $1 \mathrm{~h}$, and then in-gel-digested with sequencing grade trypsin overnight at $37^{\circ} \mathrm{C}$. Peptides were extracted with $5 \%$ formic acid $/ 50 \%$ acetonitrile and $0.1 \%$ formic acid/75\% acetonitrile sequentially and concentrated to $20 \mu$. The extracted peptides were separated by an analytical capillary column $(50 \mu \mathrm{m} \times 15 \mathrm{~cm})$ packed with $5-\mu \mathrm{m}$ spherical C18 reversed phase material (YMC, Kyoto, Japan). A Waters nanoAcquity UPLC system (Waters, Milford, USA) was used to generate the following HPLC gradient: $0-30 \% \mathrm{~B}$ in $60 \mathrm{~min}, 30-70 \% \mathrm{~B}$ in $15 \mathrm{~min}(\mathrm{~A}=0.1 \%$ formic acid in water, $\mathrm{B}=0.1 \%$ formic acid in acetonitrile). The eluted peptides were sprayed into a LTQ ORBITRAP Velso mass spectrometer (ThermoFisher Scientific, San Jose, CA, USA) equipped with a nano-ESI ion source. The mass spectrometer was operated in data-dependent mode with one MS scan followed by four CID (collision induced dissociation) and four HCD (high-energy collisional dissociation) MS/MS scans for each cycle. Database searches were performed on an in-house Mascot server (Matrix Science Ltd, London, UK) against human RHOA protein sequence. The search parameters are: 7-p.p.m. mass tolerance for precursor ions; 0.5-Da mass tolerance for product ions; three missed cleavage sites were allowed for trypsin digestion; the following variable modifications were included: oxidation on methionine, carbamidomethylation on cysteine, deamidation on asparagine or glutamine. The tandem mass spectra of matched deamidated peptides were manually checked for their validity.

Generation of $\mathbf{M e f v}^{-1-}$ mice. TALEN constructs were designed using the free online tool TAL Effector Nucleotide Targeter 2.0 (https://tale-nt.cac.cornell.edu/ node/add/talen), and assembled using the Golden Gate TALEN and TAL Effector Kit (Addgene cat. no. 1000000024), according to the published protocol ${ }^{37}$. The vector used is pCAG-T7-TALEN (Sangamo)-Destination, and the TALEN constructs were designed to target exon 2 of the mouse Mefv locus. The details of the target sequence are shown in Extended Data Fig. 4. All constructs were validated by DNA sequencing. The efficiency of the TALEN constructs was determined by transfection assay in mouse 3T 3 cells. To generate the mice, in vitro-transcribed TALEN mRNAs were injected into the cytoplasm of mouse pronuclear-stage embryos to produce mutant founders $\left(\mathrm{F}_{0}\right)$. Founders carrying frameshift mutations on one allele were intercrossed to produce the $\mathrm{F}_{1}$ generation. The T7E1 assay ${ }^{37}$ was used to validate the targeting efficiency in 3T3 cells and also screen for the desired mutant mice. To confirm the frameshift mutations, PCR products were ligated into the T-vector and more than five clones for each mouse were sent for DNA sequencing. Sequences of the mutant alleles in the five $\mathrm{F}_{1}$ mutant lines are detailed in Extended Data Fig. 4. Bacterial strains and cell culture infection. B. cenocepacia J2315 strain (obtained from the BCCM/LMG Bacteria Collection, Belgium), S. typhimurium SL1344, S. flexneri $2457 \mathrm{~T}$ and EHEC O157:H7 were cultured overnight at $37^{\circ} \mathrm{C}$ in LB broth under conditions of vigorous shaking. Bacterial cultures were diluted by 1:100 in fresh $\mathrm{LB}$ broth, and grown until $O D_{600}$ reached 0.8 . The bacteria were then diluted in serum-free RPMI-1640 medium to achieve the desired m.o.i. ( 20 for B. cenocepacia, 1 for S. typhimurium, 20 for $S$. flexneri and EHEC). Infection was started by centrifugation at $800 \mathrm{~g}$ for $10 \mathrm{~min}$ and preceded by incubation at $37^{\circ} \mathrm{C}$ for another $30 \mathrm{~min}$. Infected cells were washed three times with PBS and supplemented with fresh media containing $100 \mathrm{ng} \mathrm{ml}^{-1}$ gentamicin to kill the extracellular bacteria. $3 \mathrm{~h}$ later, the supernatants were harvested and subjected to inflammasome activation assays. $V$. parahaemolyticus ( $\Delta t d h A S \Delta v c r D 2$ POR3 strain, kindly provided by
T. Iida, Osaka University, Japan) culture and infection was performed as described previously ${ }^{38}$. To measure B. cenocepacia replication, an m.o.i. of 0.1 was used to infect primary BMDM cells. At the indicated time points after infection, macrophages were washed with PBS for three times and lysed in a buffer containing $50 \mathrm{mM}$ Tris- $\mathrm{HCl}$ (pH 7.6), $150 \mathrm{mM} \mathrm{NaCl}$ and $0.1 \%$ Triton X-100. Bacteria were recovered by plating serial dilutions of the lysates onto LB agar plates containing $100 \mu \mathrm{g} \mathrm{ml}^{-1}$ streptomycin, and colony-forming units were counted $48 \mathrm{~h}$ later. Triplicates were performed for each infection experiment and three independent experiments were carried out to confirm the results.

B. cenocepacia $\Delta h c p$ strain was first constructed by polar insertion. In brief, a PCR fragment containing flanking sequences of the $h c p$ gene was cloned into the suicide vector pDM4-SacB. The resulting targeting vector was transformed into $B$. cenocepacia through E. coli SM10 ( $\lambda$ pir)-mediated conjugational mating. The transconjugants were selected in LB agar medium containing streptomycin $\left(100 \mu \mathrm{g} \mathrm{ml}^{-1}\right)$ and chloramphenicol $\left(30 \mu \mathrm{g} \mathrm{ml}^{-1}\right)$, and positive clones containing the desired insertion were picked and verified by PCR. To obtain in-frame deletion, the pDM4-SacB vector was modified by incorporation of the yeast homing endonuclease I-SceI recognition site. The modified vector was used to obtain the polar insertion mutant as above described. The pDAI-SceI plasmid expressing I-SceI nuclease, provided by M. A. Valvano (Queen's University of Belfast, UK), was then introduced into the insertion mutant and selected for tetracyclin resistance $\left(200 \mu \mathrm{g} \mathrm{ml}^{-1}\right)$. I-SceI expression resulted in a double strand break in the B. cenocepacia chromosome and stimulation of bacterial recombination/repair machinery ${ }^{39}$. All the insertion and deletion mutants were verified by PCR and DNA sequencing.

Mice infection and histopathological analysis. For mouse infection, B. cenocepacia was cultured similarly as that for macrophage infection and prepared in PBS containing $1 \%$ gelatin. Ten-week-old male mice (C57BL/6 background) were randomized into each experimental group with no blinding. Independent experiments were performed using four mice per group. The mice were anaesthetized by intraperitoneal injection of 2,2,2-tribromethanal dissolved in 2-methyl-butanol and infected intranasally with a $30 \mu \mathrm{l}$ of the bacteria suspension $\left(1 \times 10^{8}\right.$ c.f.u.) or PBS containing $1 \%$ gelatin as the control. Mice were killed $12 \mathrm{~h}$ post-infection. For histopathological analysis, the lungs were removed en bloc and inflation-fixed via the trachea with $4 \%$ formalin overnight. Each lobe was separated and the formalin was replaced with PBS. The lungs were then soaked in $30 \%$ sucrose overnight and embedded in the OCT compound (SAKURA 4583). The lung sections (20- $\mu \mathrm{m}$ thick) were stained with haematoxylin and eosin and the images were acquired on the ZEISS AxioCamHRc microscope.

31. Gong, Y. N. etal. Chemical probing reveals insights into the signaling mechanism of inflammasome activation. Cell Res. 20, 1289-1305 (2010).

32. Zhao, Y.etal. The NLRC4 inflammasome receptors for bacterial flagellin and type III secretion apparatus. Nature 477, 596-600 (2011).

33. Yang $\mathrm{G}$. et al. Expression of recombinant Clostridium difficile toxin $A$ and $B$ in Bacillus megaterium. BMC Microbiol. 8, 192 (2008).

34. Yang, J., Zhao, Y., Shi, J. \& Shao, F. Human NAIP and mouse NAIP1 recognize bacterial type III secretion needle protein for inflammasome activation. Proc. Natl Acad. Sci. USA 110, 14408-14413 (2013).

35. Benard, V., Bohl, B. P. \& Bokoch, G. M. Characterization of Rac and Cdc42 activation in chemoattractant-stimulated human neutrophils using a novel assay for active GTPases. J. Biol. Chem. 274, 13198-13204 (1999).

36. Ren, X. D. \& Schwartz, M. A. Determination of GTP loading on Rho. Methods Enzymol. 325, 264-272 (2000).

37. Cermak, T. et al. Efficient design and assembly of custom TALEN and other TAL effector-based constructs for DNA targeting. Nucleic Acids Res. 39, e82 (2011).

38. Higa, N. et al. Vibrio parahaemolyticus effector proteins suppress inflammasome activation by interfering with host autophagy signaling. PLoS Pathog. 9, e1003142 (2013)

39. Flannagan, R. S., Linn, T.\& Valvano, M. A. A system for the construction of targeted unmarked gene deletions in the genus Burkholderia. Environ. Microbiol. 10, 1652-1660 (2008). 

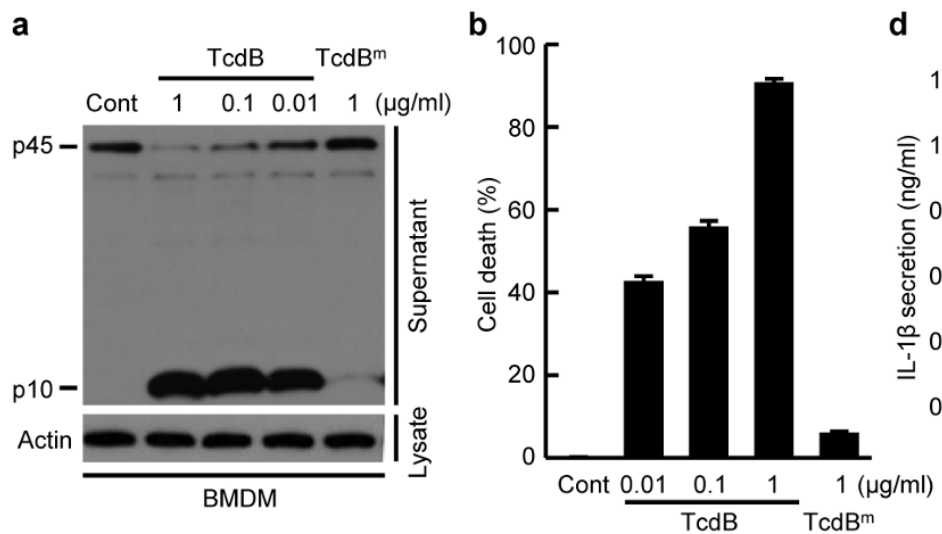

C

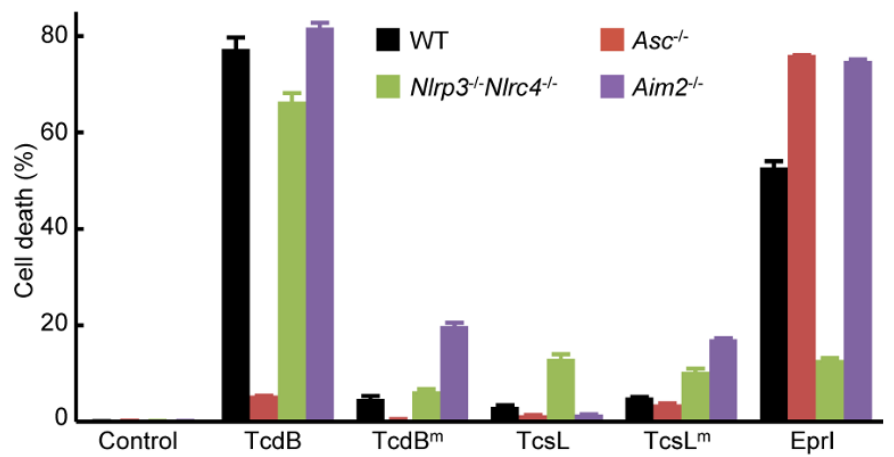

f

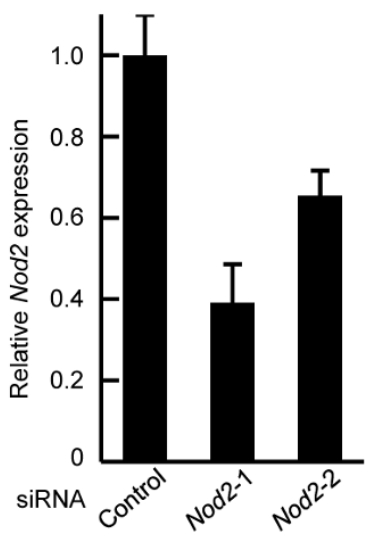

g

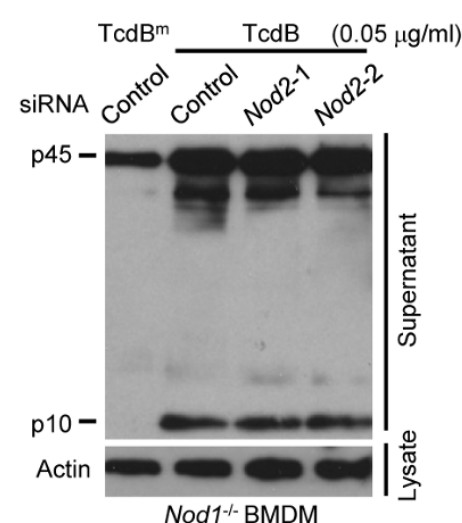

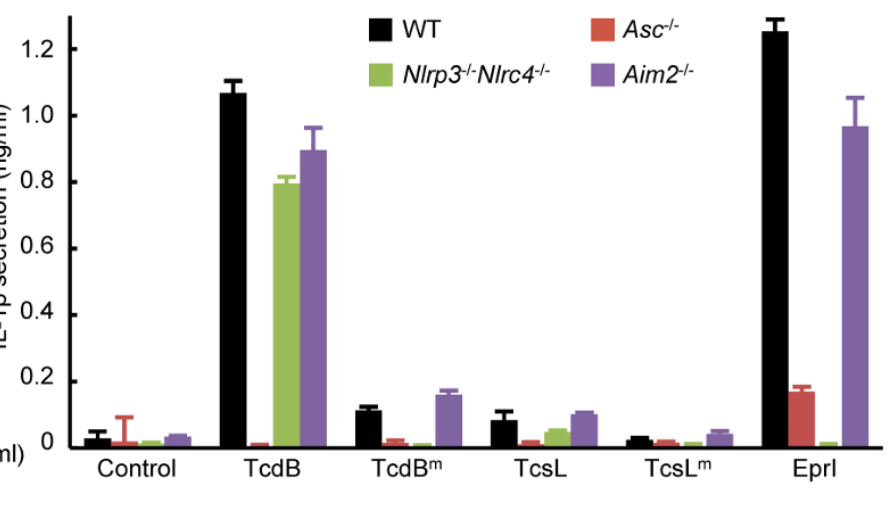

e

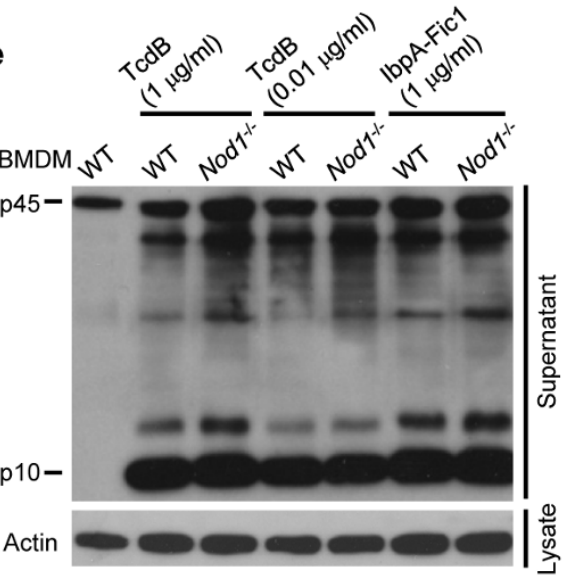

h

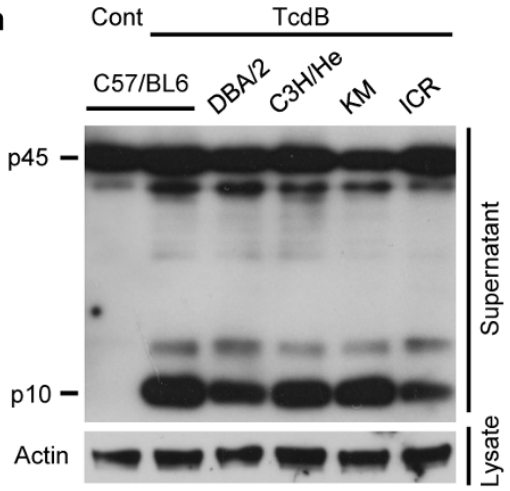

Extended Data Figure 1 TcdB-induced caspase 1 inflammasome activation in various mouse BMDMs. a-d, BMDMs from wild-type (WT, C57BL/6) or indicated knockout mice were left untreated (cont) or stimulated with TcdB, TcsL or EprI for 2.5 to $3 \mathrm{~h}$. EprI was delivered by the LFn-PA system. e-g, Wildtype or Nod1 $1^{-1-}$ BMDMs (C57BL/6 background) or Nod1 $1^{-/-}$BMDMs transfected with Nod2-specific siRNAs (Nod2-1 and Nod-2) or control siRNA were stimulated $\mathrm{TcdB}$ as indicated. The knockdown efficiency were measured by qRT-PCR analyses in $\mathbf{f}(n=3$; mean \pm s.d.). $\mathbf{h}, \mathrm{BMDMs}$ from different mouse inbred strains were stimulated with $\mathrm{TcdB}$. $\mathrm{TcdB}^{\mathrm{m}}$ and $\mathrm{TcsL}^{\mathrm{m}}$ denote the glucosyltransferase-deficient TcdB(W102A/D288N) and TcsL(D286N/ D288N) mutants, respectively. Macrophage supernatants were collected for anti-caspase 1 immunoblotting analyses in $\mathbf{a}, \mathbf{e}, \mathbf{g}$ and $\mathbf{h}$ (representative of at least three repetitions). ELISA assay of IL- $1 \beta$ release in LPS-primed BMDMs is shown in $\mathbf{d}$ and percentages of cell death measured by LDH (lactate dehydrogenase) release are in $\mathbf{b}, \mathbf{c}(n=3$; mean \pm s.d.). 
a
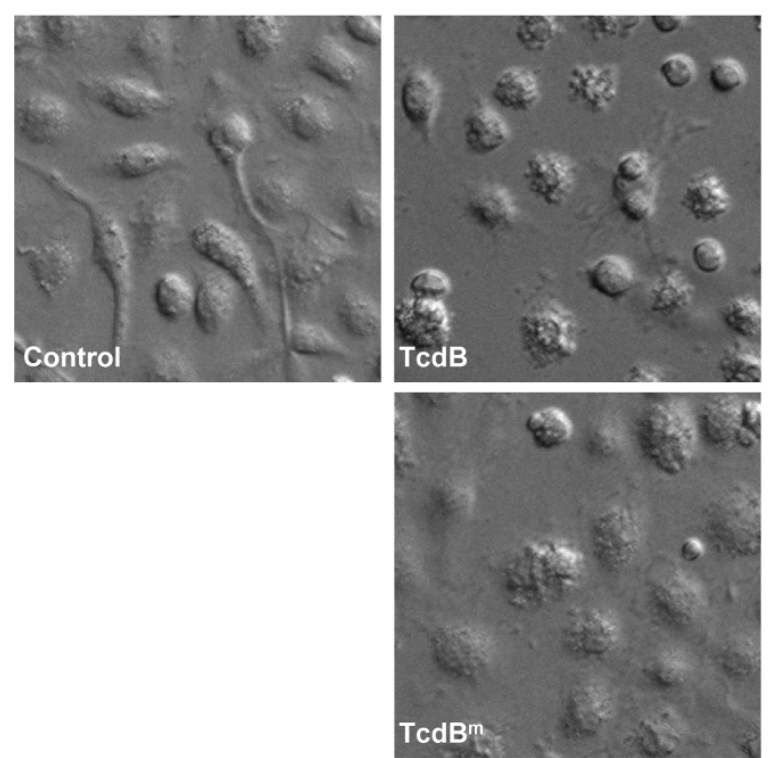

b
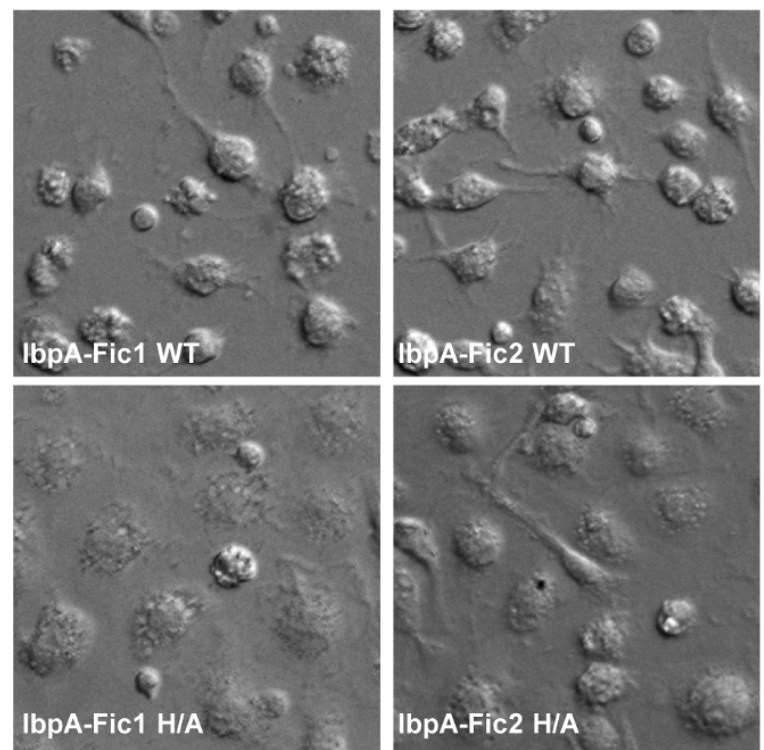
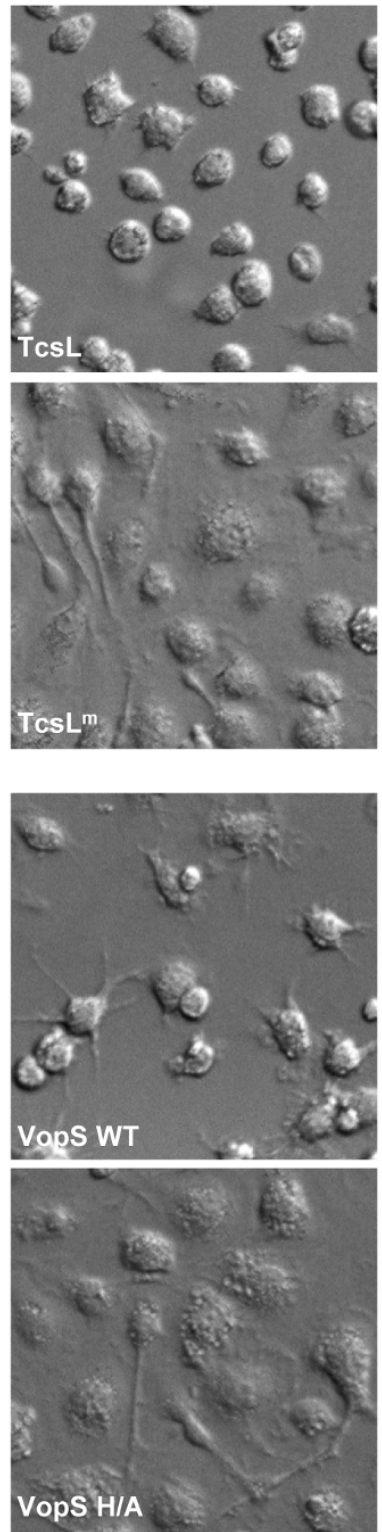

Extended Data Figure $2 \mid$ Cell rounding induced by Rho-modifying toxins and effectors. Caspase $1^{-1-}$ BMDMs were left untreated (control) or stimulated with large clostridial glucosylating cytotoxins $\mathrm{TcdB} / \mathrm{TcsL}$ in $\mathbf{a}$ and FIC-domain bacterial effectors (VopS and IbpA-Fic1/2) in b. TcdB ${ }^{\mathrm{m}}$ and $\mathrm{TcsL}^{\mathrm{m}}$ denote the corresponding catalytically inactive mutants. VopS, IbpA-Ficl and IbpA-Fic2 (WT or the catalytically inactive H/A mutants) were delivered into BMDMs using the LFn-PA system. Representative differential interference contrast (DIC) microscopy images of cell morphology are shown. 

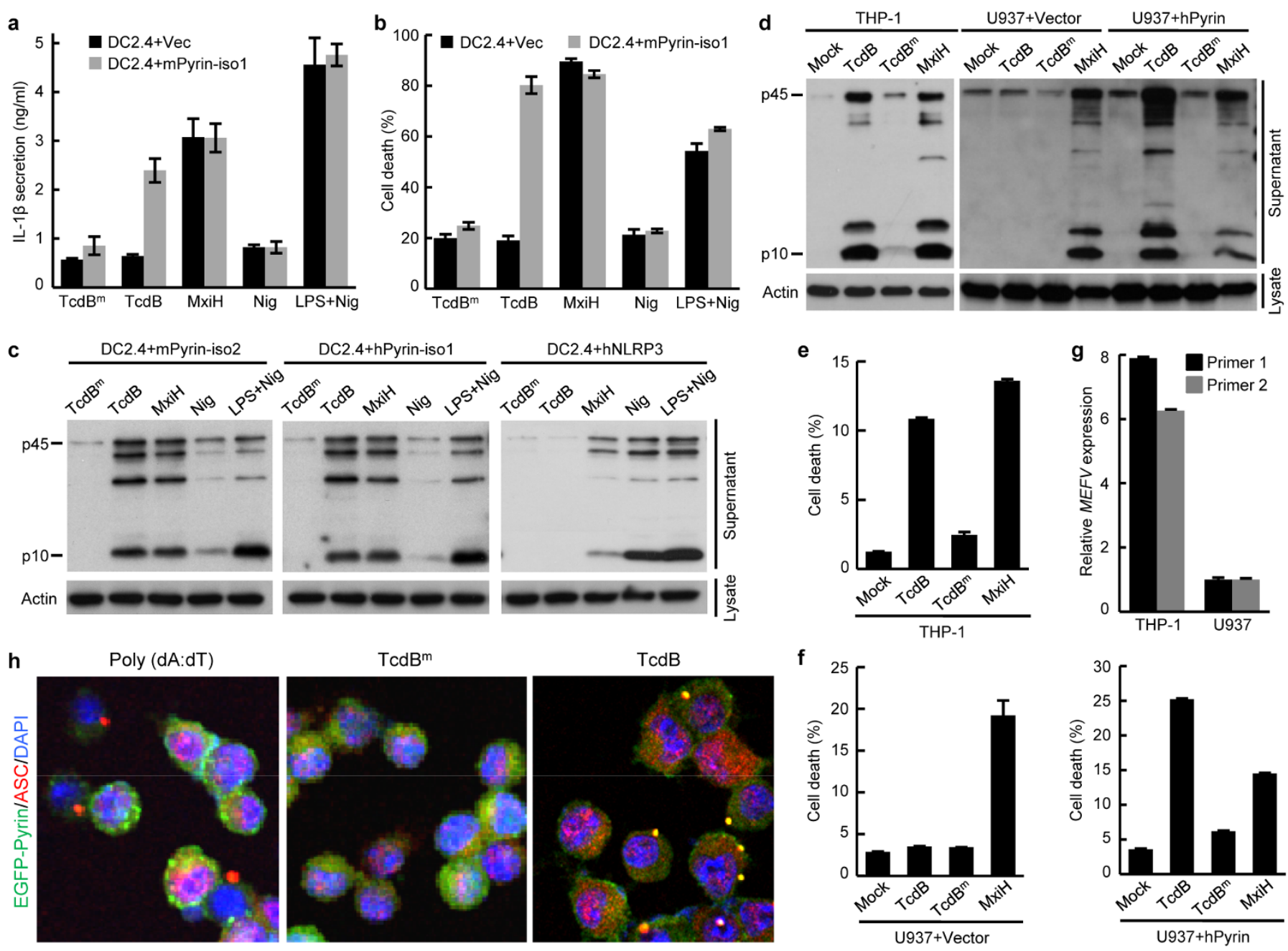

Extended Data Figure 3 TcdB-induced caspase 1 inflammasome activation in Pyrin-complemented DC2.4 cells and human monocyte-derived macrophages. a-c, DC2.4 cells harbouring a vector (DC2.4+Vec), mouse Pyrin isoform 1 (DC2.4+mPyrin-isol), mouse Pyrin isoform 2

(DC2.4+ mPyrin-iso2), human Pyrin isoform 1 (DC2.4+hPyrin-iso1) or NLRP3 were stimulated with TcdB or as indicated. $\mathbf{d}-\mathbf{f}$, Phorbol-12-myristate13-acetate (PMA)-differentiated THP-1 or U937 cells (harbouring a vector or human Pyrin isoform 1) were stimulated with TcdB or MxiH. g, qRT-PCR measurements of relative Pyrin expression level (normalized to that of GAPDH) ( $n=3$; mean \pm s.d.). h, DC2.4 cells stably expressing eGFP-Pyrin were stimulated with $\mathrm{TcdB}$ or poly(dA:dT), and then subjected to anti-ASC immunofluorescence staining. DAPI stains the nuclei. The merged images show the co-aggregation of eGFP-Pyrin with endogenous ASC. Representative caspase 1 immunoblots from at least three repetitions are shown in $\mathbf{c}$ and $\mathbf{d}$. ELISA assay of IL- $1 \beta$ release in $\mathbf{a}$ and percentages of cell death measured by $\mathrm{LDH}$ release in $\mathbf{b}$, $\mathbf{e}$ and $\mathbf{f}$ are mean \pm s.d. $(n=3)$. 
a

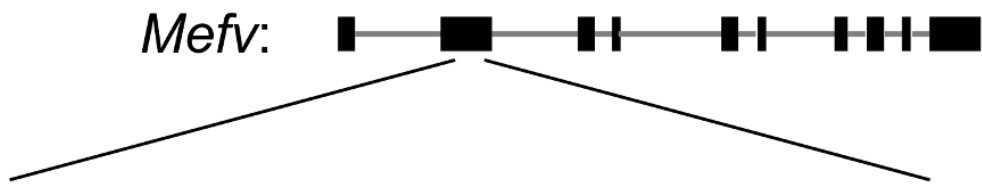

NN NI NG NN NI NI NG HD NI NN NI HD NI HD HD
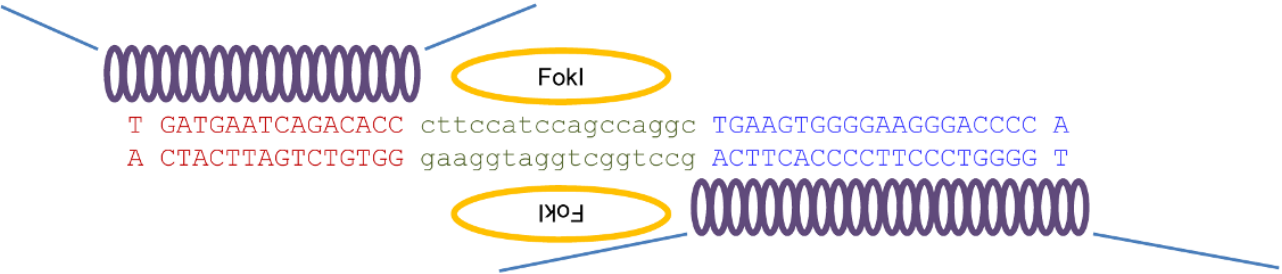

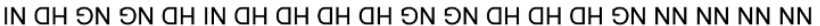

b

$$
\begin{array}{llllllllllll}
T & \mathrm{~L} & \mathrm{P} & \mathrm{S} & \mathrm{S} & \mathrm{Q} & \mathrm{A} & \mathrm{E} & \mathrm{V} & \mathrm{G} & \mathrm{K} & \mathrm{G}
\end{array}
$$

F1-1 ACC CTT CC- $-\ldots \ldots-\ldots$ GCT GAA GTG GGG AAG GGA

F1-1 ACC CTT CCA TC- -- -AG GCT GAA GTG GGG AAG GGA

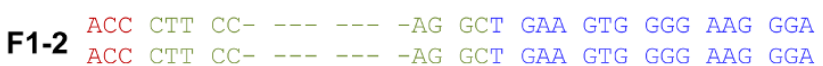

11 ACC CTT CC- $-\ldots-\ldots-$ AG GCT GAA GTG GGG AAG GGA

F1-3 ACC CTT CCA TC- --- AG GCT GAA GTG GGG AAG GGA

F1-4 ACC CTT CC- $---\cdots-$ AG GCT GAA GTG GGG AAG GGA

F1-5 ACC CTT CC- --- -- - AG GCT GAA GTG GGG AAG GGA

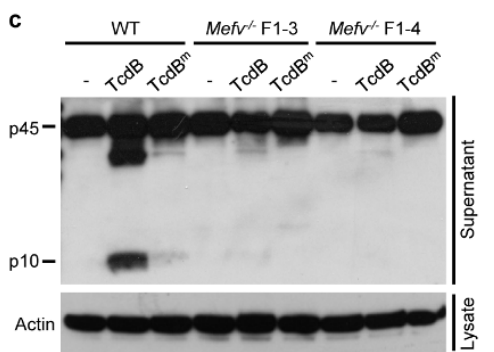

Extended Data Figure $4 \mid$ TALEN-mediated Mefv knockout in mice and its effect on TcdB-induced caspase 1 activation. a, The design of Mefvtargeting TALEN. $\mathbf{b}$, The sequence mutations for the five homozygous $\mathrm{F}_{1}$ lines used in the study. $\mathrm{F}_{1}-1,3$ and 4 were obtained by intercross of two heterozygous founders bearing different frameshift alleles. $\mathrm{F}_{1}-2$ and 5 were crossed from another two heterozygous founders harbouring the same frameshift allele. c, BMDMs from Mefv ${ }^{-/-} \mathrm{F}_{1}-3$ and $\mathrm{F}_{1}-4$ mice were stimulated with TcdB or $\mathrm{TcdB}^{\mathrm{m}}$. Shown are representative caspase 1 immunoblots from at least three repetitions. 


\section{RESEARCH LETTER}
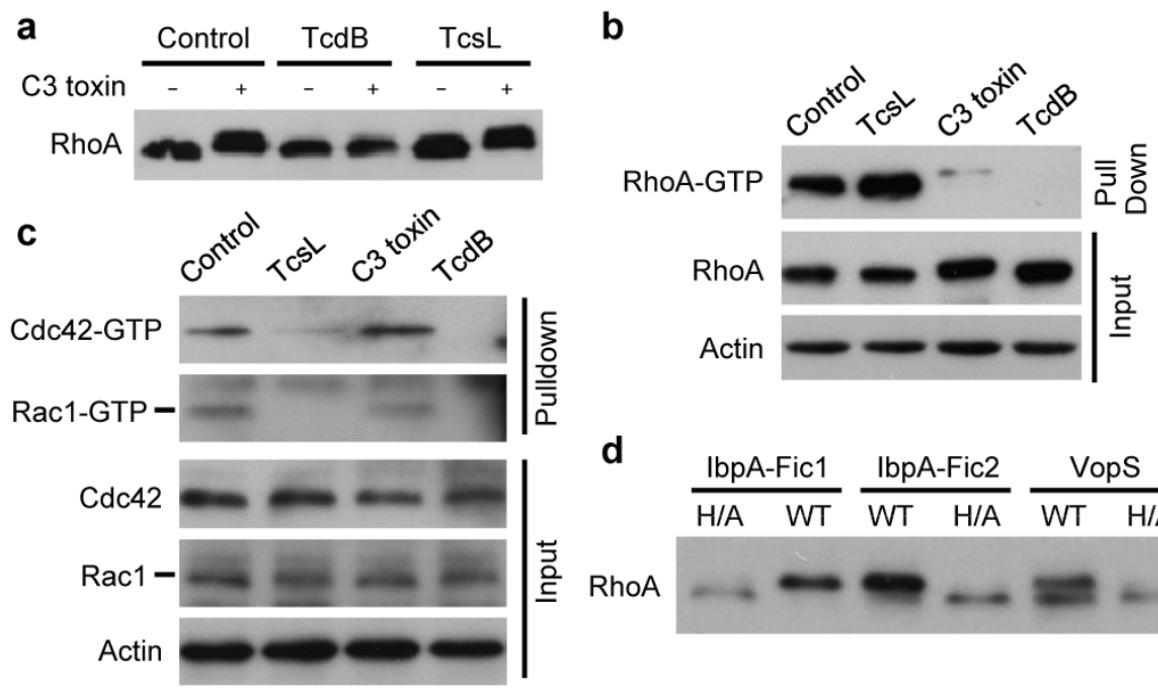

d

b

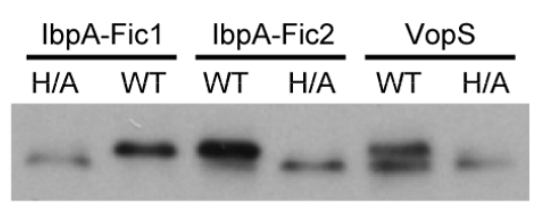

e

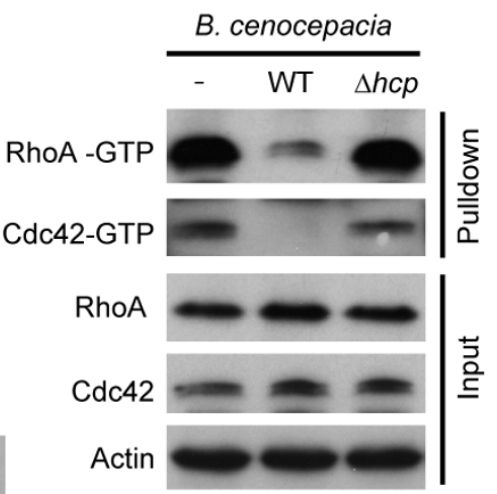

Extended Data Figure 5 Toxins-induced modification of Rho GTPases and Rho inactivation by TcdB, TcsL, C3 and $B$. cenocepacia infection. a, Lysates of TcdB or TcsL-treated Caspase $1^{-/-}$BMDM cells were subjected to in vitro ADP-ribosylation reaction by purified $\mathrm{C} 3$ toxin. Anti-RHOA immunoblotting shows TcdB modification of RHOA, as suggested by its resistance to further modification by the $\mathrm{C} 3$ toxin. $\mathbf{b}, \mathbf{c}, \mathbf{e}, \mathrm{DC} 2.4$ cells were stimulated with TcdB, TcsL or LFn-tagged C3 toxin in $\mathbf{b}, \mathbf{c}$, or infected with B. cenocepacia (WT or the $\Delta h c p$ mutant) in e. Cell lysates were subjected to glutathione $S$-transferase (GST)-RBD (the Rho binding domain of human
Rhotekin protein) (b,e) and GST-PBD (the Rac/Cdc42 (p21) binding domain of human $\mathrm{p} 21$ activated kinase 1 protein $(\mathbf{c}, \mathbf{e})$ pulldown assays to measure GTP-bound RHOA and Rac/Cdc42, respectively. d, Caspase $1^{-/-}$BMDMs were delivered with $V$. parahaemolyticus VopS and the two FIC domains in H. somni IbpA (IbpA-Fic1/2) by the LFn-PA system. H/A, mutation of the FIC-domain catalytic histidine. Anti-RHOA immunoblotting shows the SDS-PAGE mobility shift of RHOA as a result of effector-catalysed adenylylation. Data in all panels are representative of at least three repetitions. 


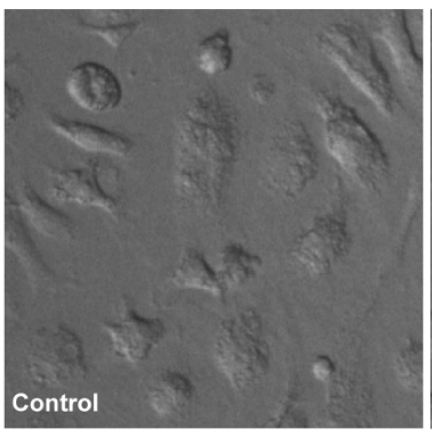

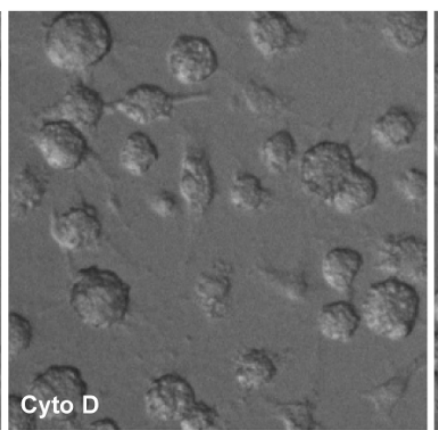

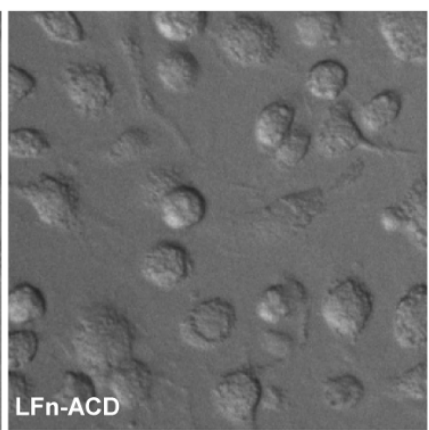

b

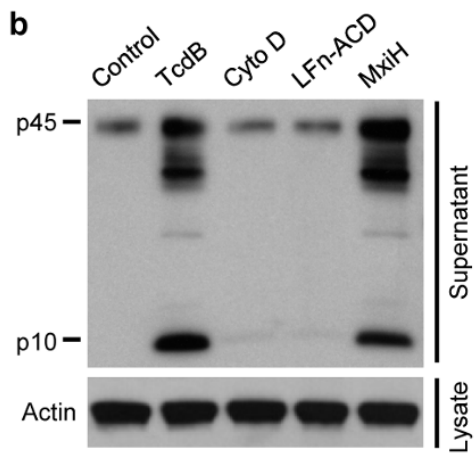

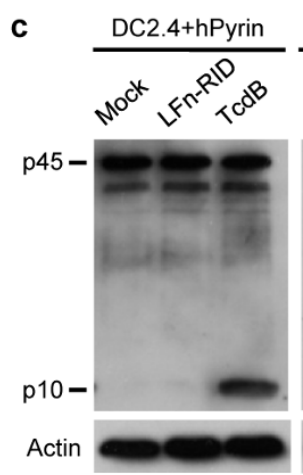

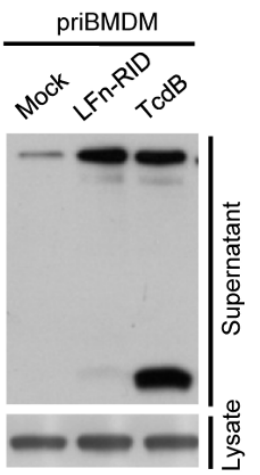

e
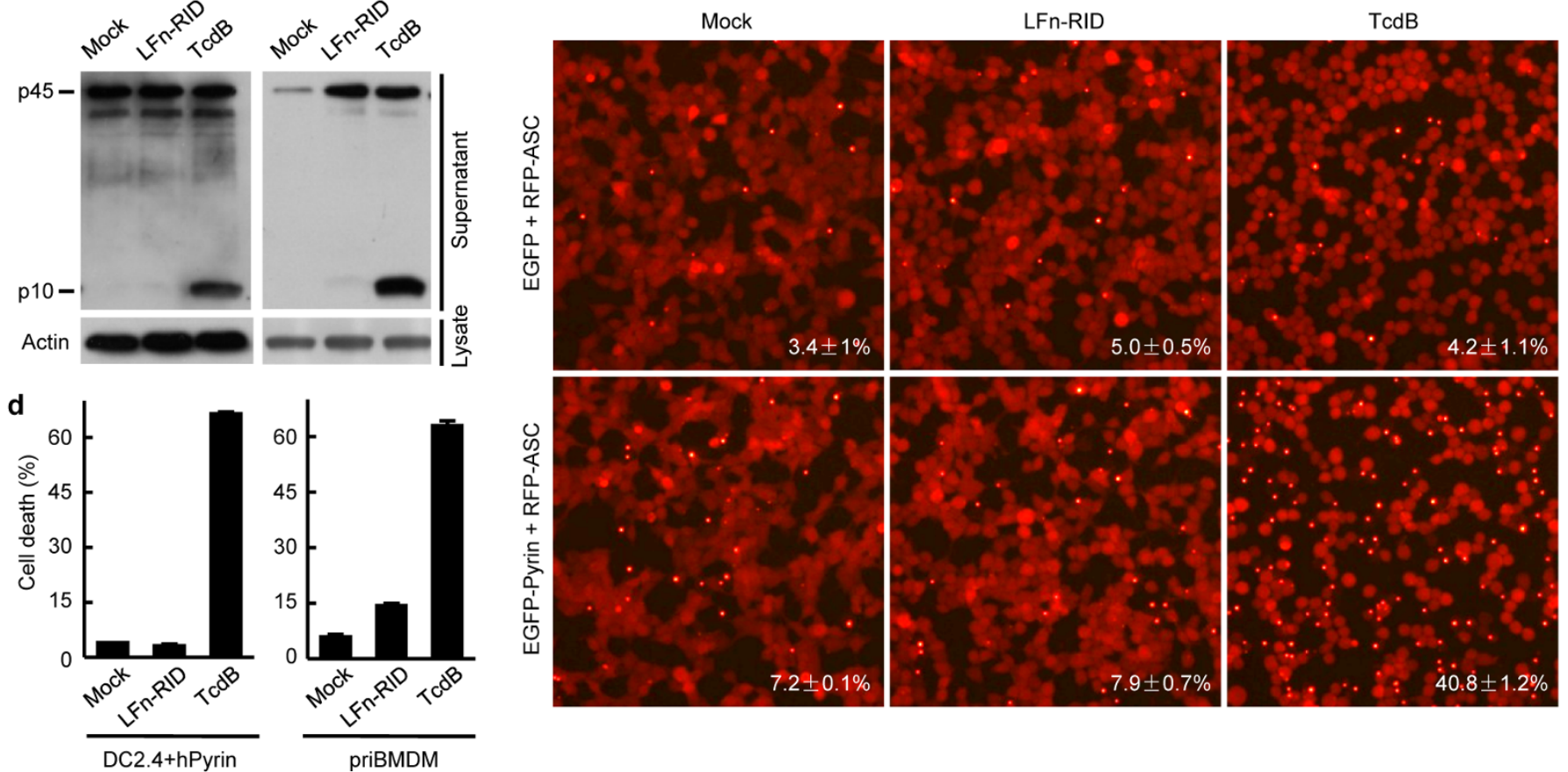

Extended Data Figure 6 | Actin polymerization-inhibiting agents and the RID domain of Vibrio RTX toxin cannot activate caspase 1 inflammasome. a, b, Primary BMDMs were left untreated (control), treated with cytochalasin D (Cyto D) or delivered with LFn-tagged actin crosslinking domain (ACD) from Vibrio RTX toxin. c-e, Primary BMDMs or Pyrin-complemented DC2.4 or 293T cells were stimulated with LFn-tagged RID domain from $V$. cholerae RTX

toxin or TcdB. Representative DIC microscopy images of cell morphology are shown in $\mathbf{a}$. Caspase 1 immunoblots are shown in $\mathbf{b}$ and $\mathbf{c}$; percentages of cell death measured by $\mathrm{LDH}$ release are in $\mathbf{d}(n=3$; mean \pm s.d.). Fluorescence images of RFP-ASC stably expressed in $293 \mathrm{~T}$ cells are presented in $\mathbf{e}$. Data in all panels are representative of at least three repetitions. 
a

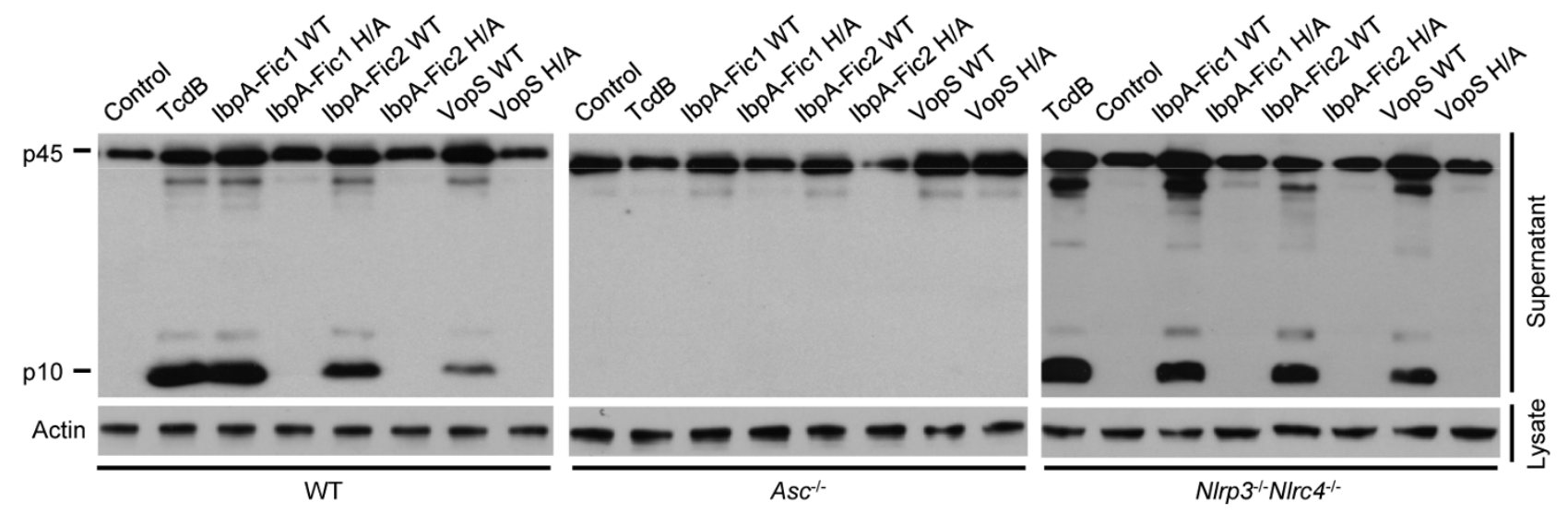

b

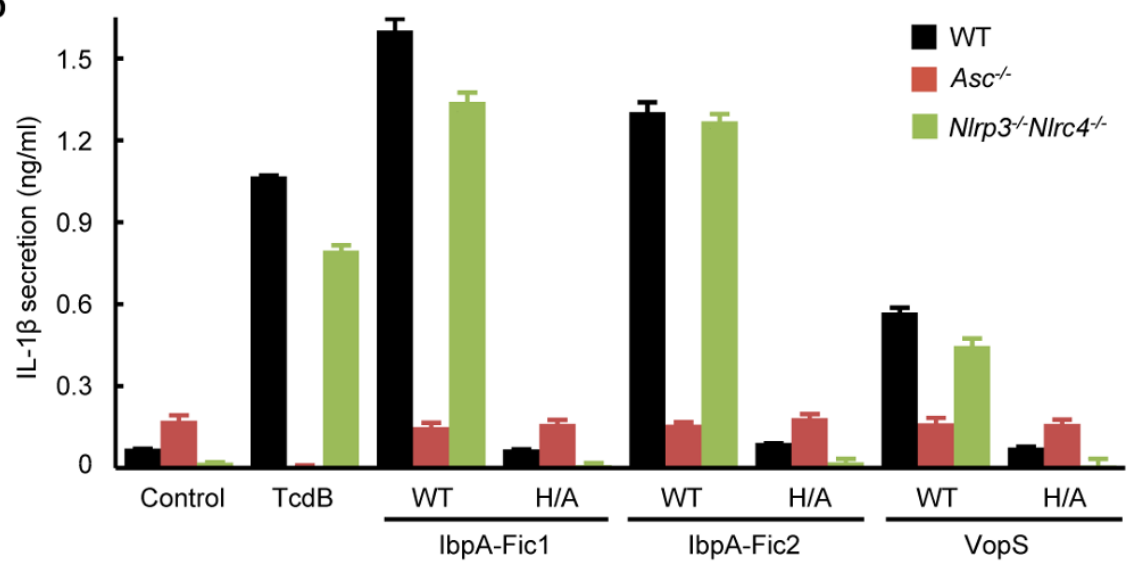

d

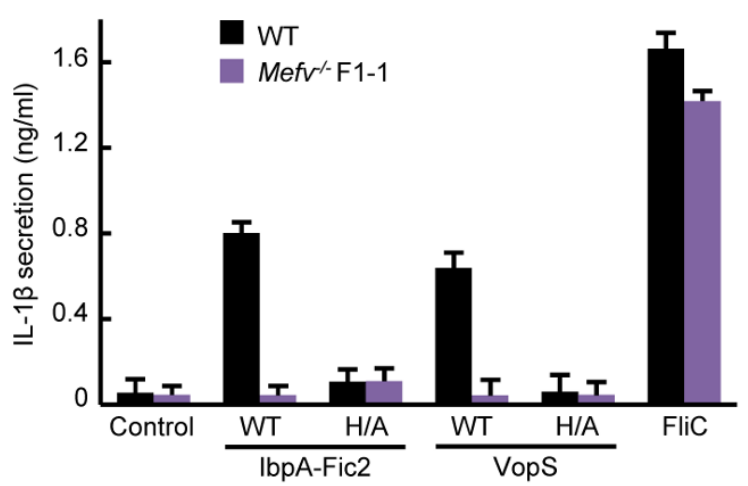

Extended Data Figure $7 \mid$ Pyrin inflammasome activation by FIC-domain Rho-adenylylating effectors. a, b, Effects of Nlrp3, Nlrc4 and Asc knockout on inflammasome activation by FIC-domain Rho-adenylylating effectors. c, d, BMDMs from wild-type mice or two independent $\mathrm{Mefv}^{-1-}$ lines $\left(\mathrm{F}_{1}-1\right.$ and $\left.\mathrm{F}_{1}-5\right)$ were stimulated with various FIC-domain effectors. e, f, Pyrincomplemented DC2.4 cells or primary BMDMs from wild-type or Mefv ${ }^{-/-}$
C

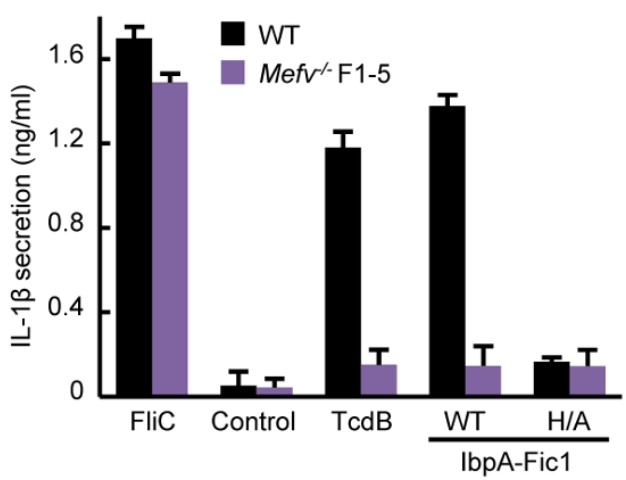

e EGF-Pyrin Vec

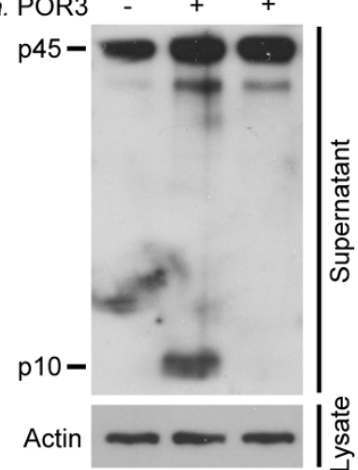

f

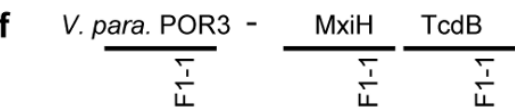

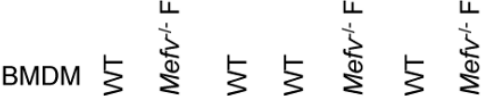

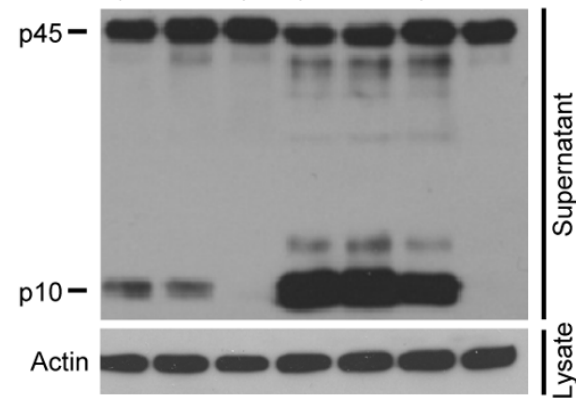

$\mathrm{F}_{1}-1$ mice were stimulated with indicated toxins or infected with V. parahaemolyticus (V.para) POR3 strain ( $\Delta t d h A S \Delta v c r D 2)$. VopS and IbpAFic1/2 were delivered into indicated BMDMs by the LFn-PA system. H/A, mutation of the catalytic histidine. Representative caspase 1 immunoblots from at least three repetitions are shown in $\mathbf{a}, \mathbf{e}$ and $\mathbf{f}$. ELISA assay of IL- $1 \beta$ release is in $\mathbf{b}, \mathbf{c}, \mathbf{d}(n=3$; mean \pm s.d. $)$. 
a

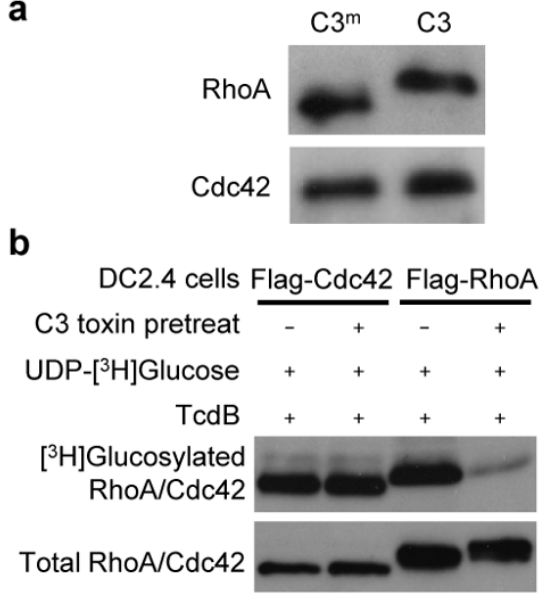

C
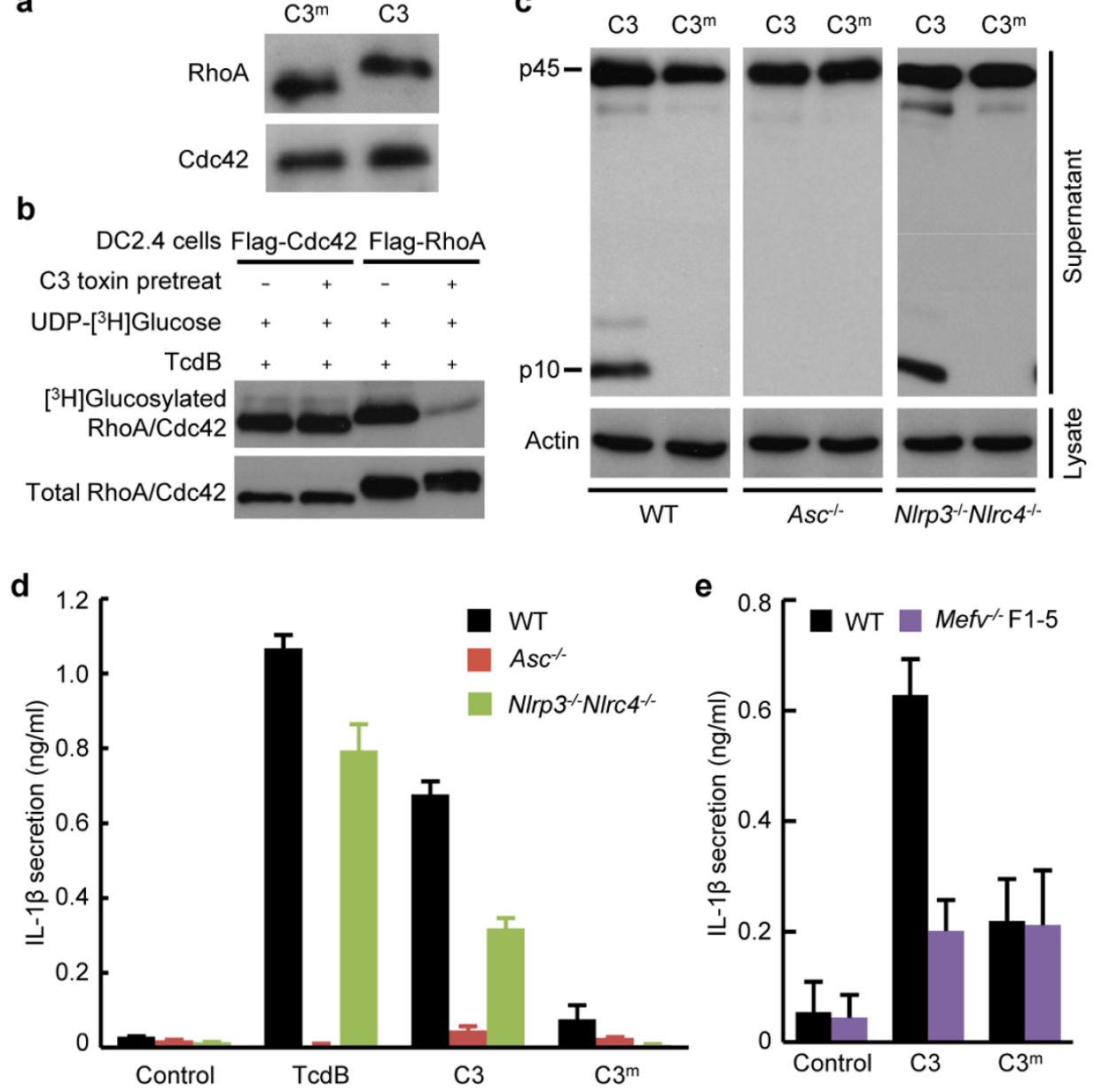

Extended Data Figure $8 \mid$ C3 modification/inactivation of the Rho subfamily induces Pyrin inflammasome activation. C3 toxin was delivered into indicated BMDMs in a, c-e or DC2.4 cells stably expressing Flag-RHOA/ Cdc42 in b by using the LFn-PA system. The SDS-PAGE mobility shift in anti-RHOA/Cdc42 immunoblots in a shows the specific modification of RHOA by C3. Flag-RHOA/Cdc42 purified from DC2.4 cells was subjected to
TcdB glucosylation in the presence of UDP- $\left[{ }^{3} \mathrm{H}\right]$ Glucose. ${ }^{3} \mathrm{H}$-autoradiograph and anti-Flag immunoblot in $\mathbf{b}$ show that $\mathrm{C} 3$ stimulation blocks $\mathrm{TcdB}$ modification of RHOA but not Cdc42. Caspase 1 immunoblots are shown in c; ELISA assay of IL- $1 \beta$ release in LPS-primed BMDMs is in $\mathbf{d}$ and $\mathbf{e}(n=3$; mean \pm s.d.). Data in all panels are representative of at least three repetitions. 


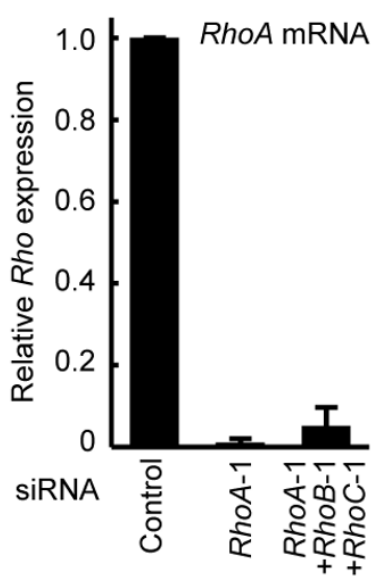

C

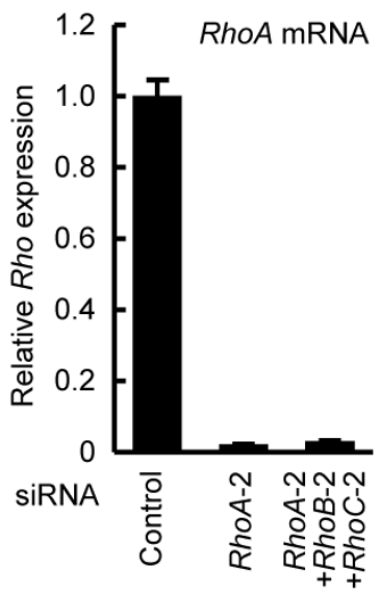

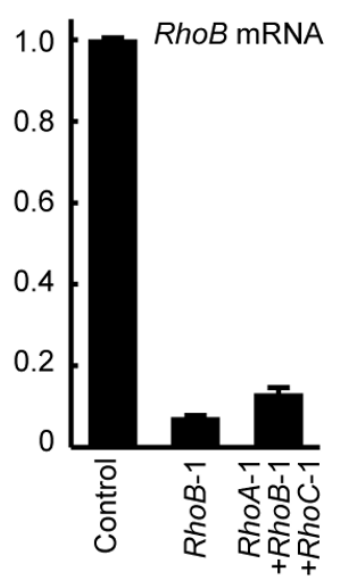
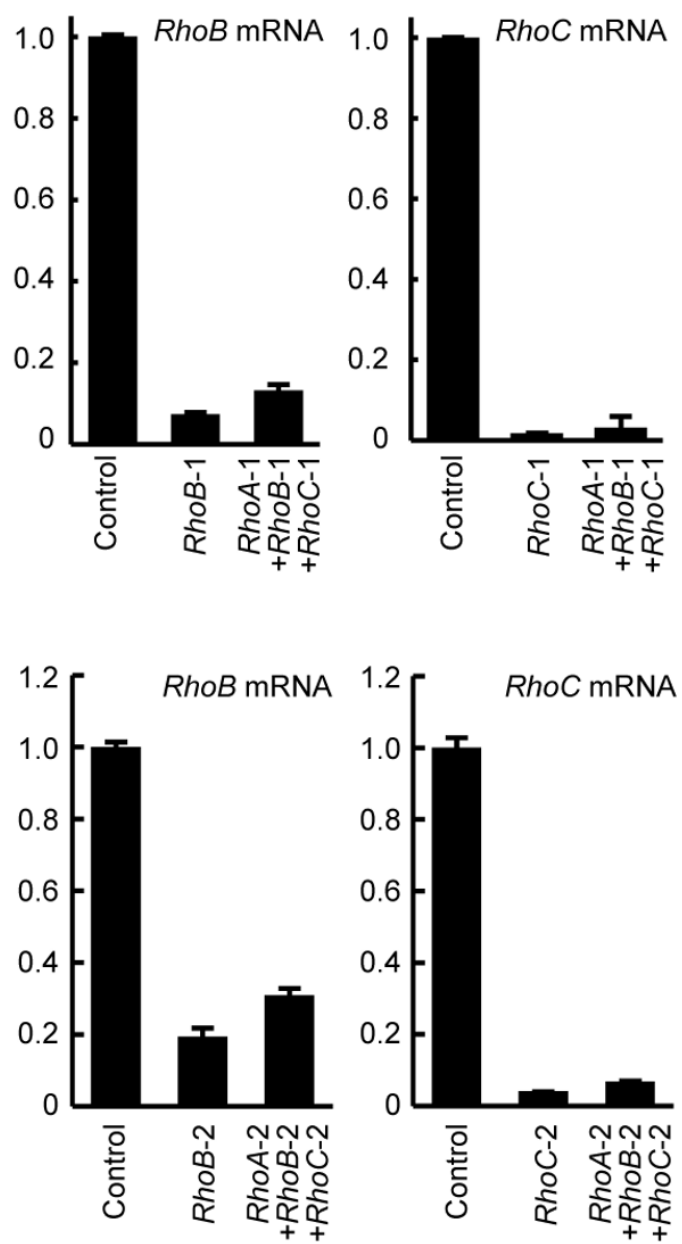

b

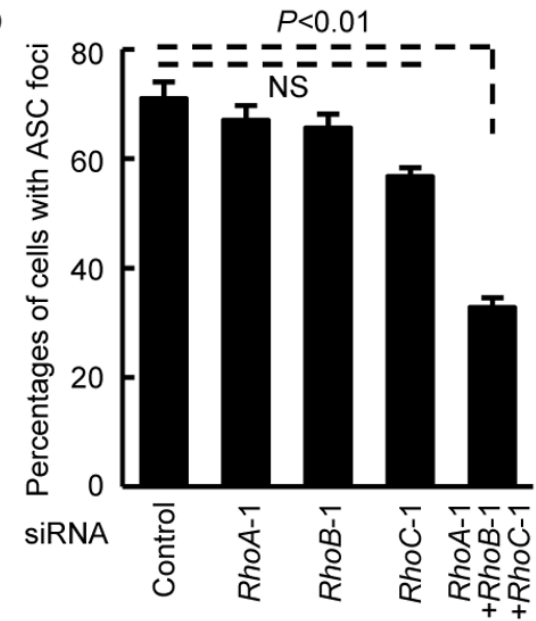

d

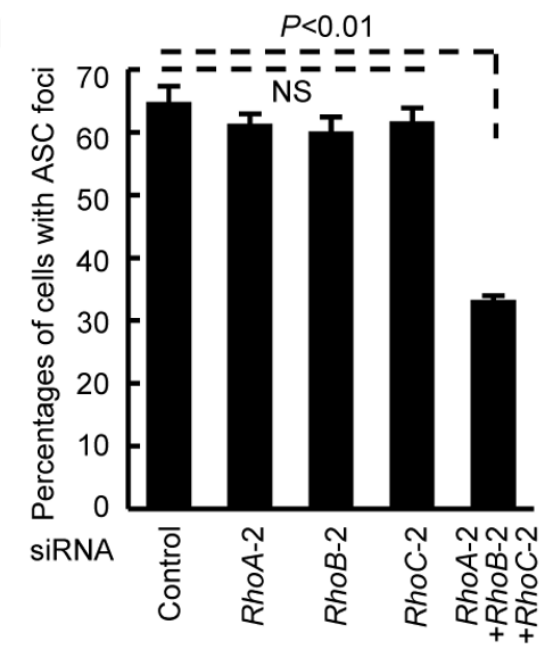

e

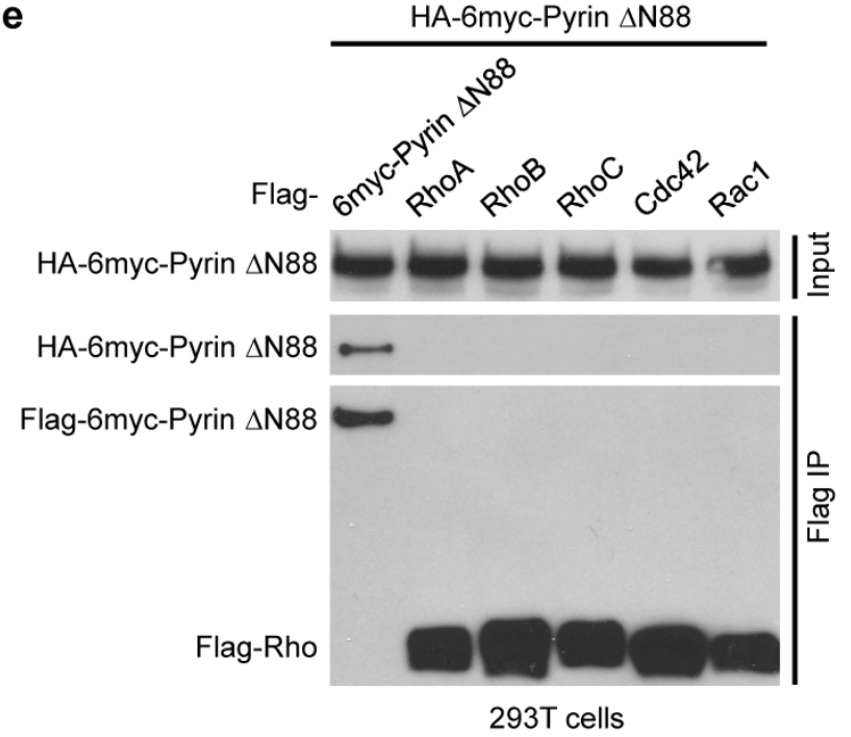

f

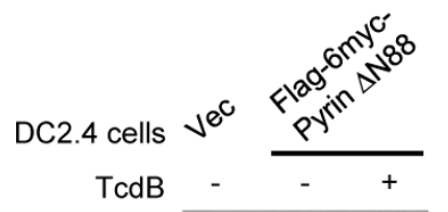

Flag-6mycPyrin $\triangle \mathrm{N} 88$

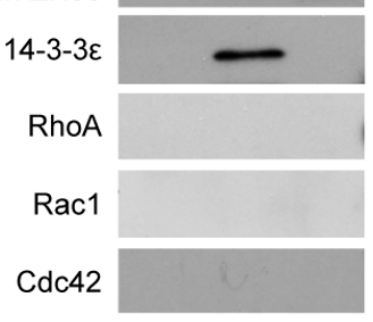

Flag IP

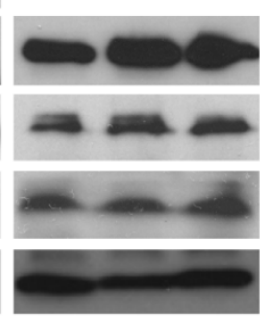

Input
Extended Data Figure $9 \mid$ Pyrin requires, but does not directly interact with, the Rho subfamily in mediating TcdB-induced inflammasome activation. a-d, Effects of siRNA knockdown of RHOA, B and C on TcdB-induced ASC foci formation. 293T cells stably expressing Pyrin and RFP-ASC were transfected with indicated siRNA or siRNA combinations followed by TcdB stimulation. Rhoa-1/2, Rhob-1/2 and Rhoc-1/2 are two independent siRNAs targeting RHOA, B and C, respectively. qRT-PCR analyses of the knockdown efficiency are shown in $\mathbf{a}, \mathbf{c}(n=3$; mean \pm s.d.) and percentages of cells showing ASC foci formation are in $\mathbf{b}, \mathbf{d}(n=3$, mean \pm s.d.; $P$ value, Student's t-test). e, f, Co-immunoprecipitation interaction assays of Pyrin and Rho GTPases. 293T cells were transfected with haemagglutinin (HA)-6mycPyrin $\Delta$ N88 (deletion of N-terminal 88 residue.) together with Flag-6mycPyrin $\Delta$ N88 or an indicated Rho GTPase construct in e. DC2.4 cells stably expressing Flag-6myc-Pyrin $\Delta$ N88 were left untreated or stimulated with $\mathrm{TcdB}$ in $\mathbf{f}$. Immunoblots of anti-Flag immunoprecipitates (Flag IP) and total cell lysates (input) shown in $\mathbf{e}$ and $\mathbf{f}$ are representative from at least three repetitions. 


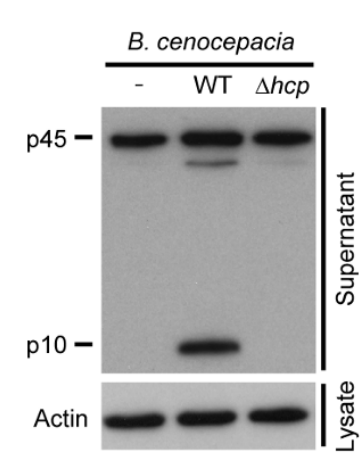

b

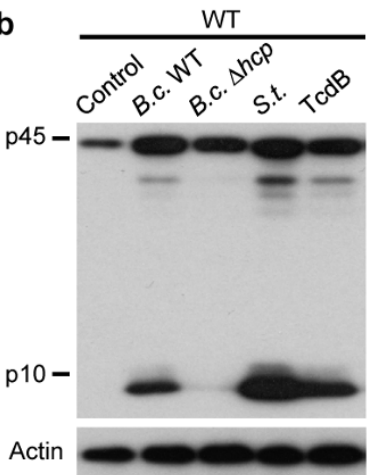

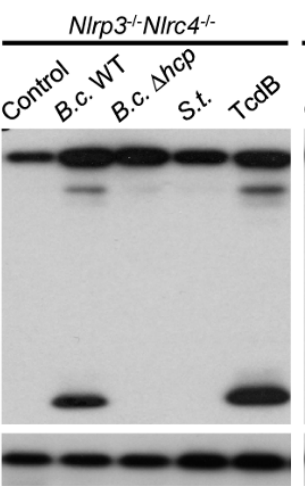

e

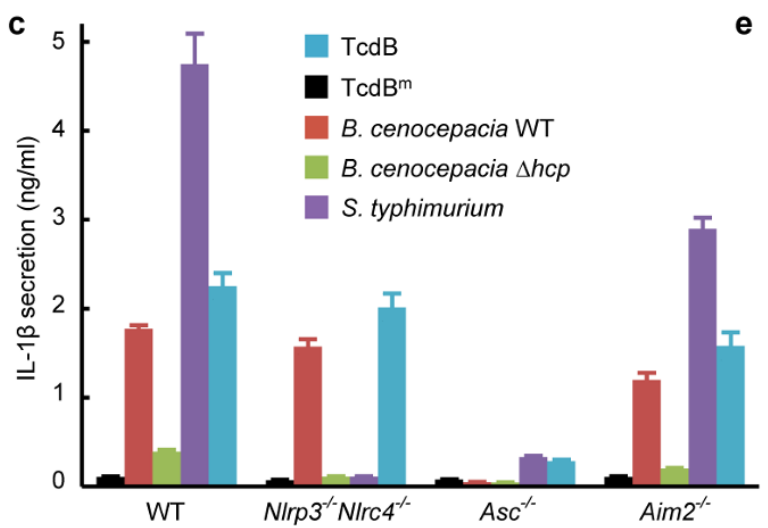

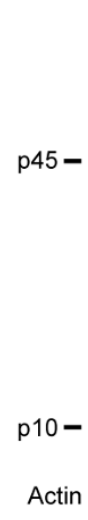
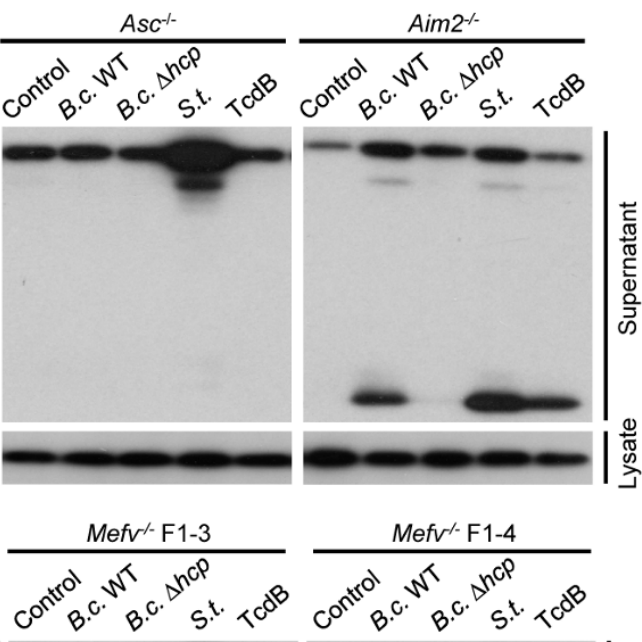

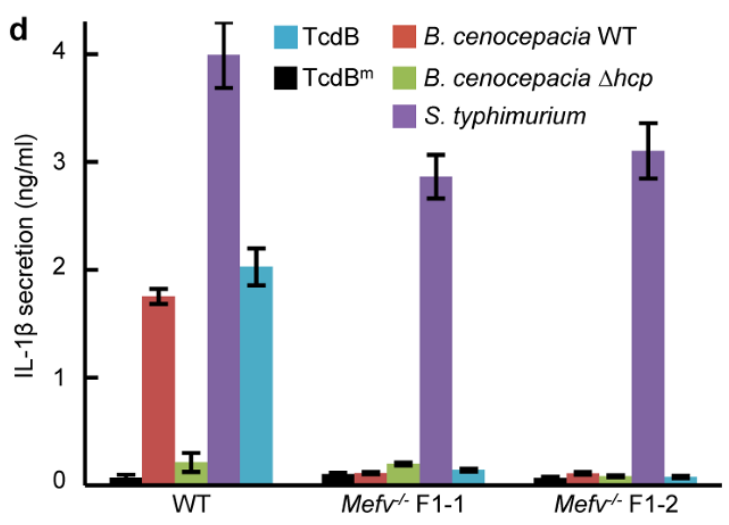

f

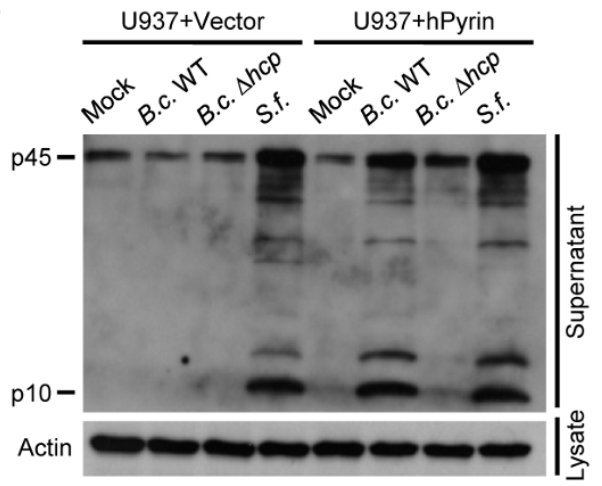

h
$\mathrm{p} 45-$
$\mathrm{p} 10-$
Actin<smiles>[10BH2]C</smiles>
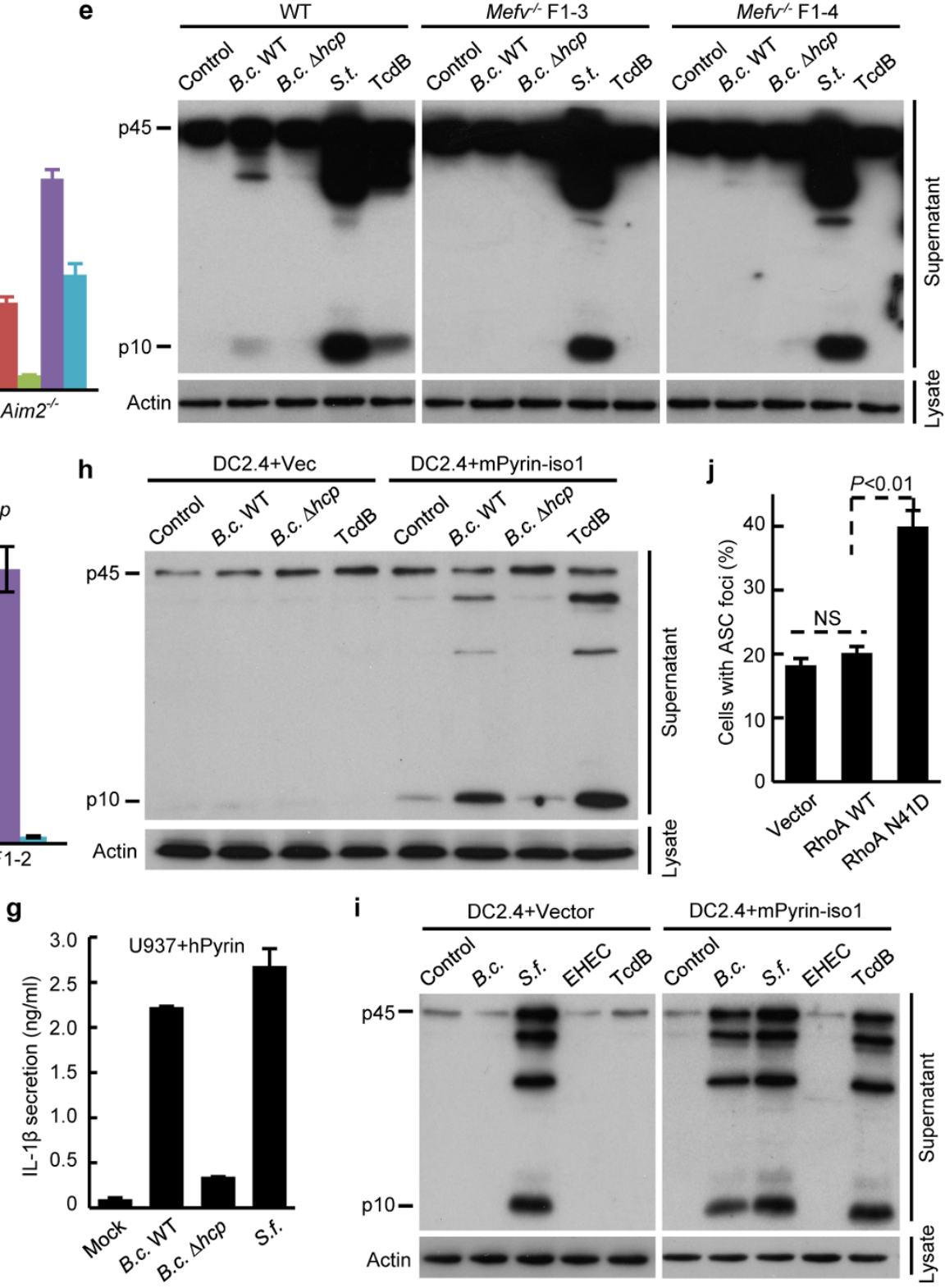

Extended Data Figure $10 \mid$ Pyrin senses B. cenocepacia-induced Asn 41 deamidation of RHOA and induces caspase 1 inflammasome activation in the T6SS-dependent manner. a-e, Wild-type, $\mathrm{Nlrp}^{-1-} \mathrm{NlrC4}{ }^{-1-}, \mathrm{Aim}^{-/-}$, $A s c^{-1-}$ and $\mathrm{Mefv}^{-1-}$ BMDMs were infected with B. cenocepacia $\mathrm{J} 2315$ strain (B.c., wild-type or the $\Delta h c p$ mutant) or S. typhimurium (S.t.) for $3 \mathrm{~h}$ at a multiplicity of infection (m.o.i.) of 20:1. f-i, U937 or DC2.4 cells harbouring a vector or expressing human Pyrin (hPyrin) or mouse Pyrin isoform 1 (mPyriniso1) were infected with B. cenocepacia (B.c.) (wild type or the $\Delta h c p$ mutant),
S. flexneri (S.f.), EHEC, or stimulated with TcdB as indicated. $\mathbf{j}$, Effects of overexpression of deamidated RHOA on ASC foci formation. The RHOA, B and C triple-knockdown $293 \mathrm{~T}$ cells obtained in Fig. 4e were transiently transfected with an empty vector or a plasmid overexpressing RHOA wild type or the N41D mutant. Representative caspase 1 immunoblot from at least three repetitions are shown in $\mathbf{a}, \mathbf{b}, \mathbf{e}, \mathbf{f}, \mathbf{h}$ and $\mathbf{i}$. ELISA assay of IL- $1 \beta$ release in $\mathbf{c}, \mathbf{d}$ and $\mathbf{g}$ and percentages of cells showing the ASC foci in $\mathbf{j}$ are mean \pm s.d. $(n=3)$. For $\mathbf{j}$, the $P$ value was determined by Student's $t$-test. 